\title{
HILBERT TRANSFORMS AND MAXIMAL FUNCTIONS ASSOCIATED TO FLAT CURVES ON THE HEISENBERG GROUP
}

\author{
ANTHONY CARBERY, STEPHEN WAINGER, AND JAMES WRIGHT
}

\section{INTRODUCTION}

Suppose for each $x$ in $\mathbb{R}^{n} \gamma(x, t)$ is a smooth curve in $\mathbb{R}^{n}$ with $\gamma(x, 0)=$ $x$. For $f \in C_{0}^{\infty}\left(\mathbb{R}^{n}\right)$, we define the Hilbert transform and maximal function associated to $\gamma(x, t)$ as

$$
H f(x)=\text { p.v. } \int_{-1}^{1} f(\gamma(x, t)) \frac{d t}{t}
$$

and

$$
\mathscr{M} f(x)=\sup _{0<h \leq 1} \frac{1}{h} \int_{0}^{h}|f(\gamma(x, t))| d t
$$

respectively.

We are interested in $L^{p}$ estimates for $H f$ and $\mathscr{M} f$. If $\gamma(x, t)$ satisfies an appropriate curvature condition then

$$
\|H f\|_{L^{p}} \leq A_{p}\|f\|_{L^{p}}, \quad 1<p<\infty,
$$

and

$$
\|\mathscr{M} f\|_{L^{p}} \leq A_{p}\|f\|_{L^{p}}, \quad 1<p \leq \infty .
$$

See [C1] and [CNSW].

We are interested in obtaining estimates (1) and (2) above for curves $\gamma(x, t)$ for which the curvature condition fails. There are a number of papers dealing with this question if $\gamma(x, t)$ is of the form

$$
\gamma(x, t)=x+\Gamma(t)
$$

where $\Gamma(t)$ is a fixed curve. See [CVWWA] or [CZ], for example.

In this paper we shall consider certain curves $\gamma(x, t)$ which are not of the form (3). In fact these curves will be curves on the Heisenberg group, that is we take a fixed curve $\Gamma(t)$ in $\mathbb{R}^{3}$, and for $x$ in $\mathbb{R}^{3}$ we set

$$
\gamma(x, t)=x \cdot \Gamma^{-1}(t)
$$

Received by the editors September 22, 1993.

1991 Mathematics Subject Classification. Primary 42B20, 42B25.

The first and third authors were supported in part by an SERC grant.

The second author was supported in part by an NSF grant. 
where $\Gamma^{-1}(t)=-\Gamma(t)$, and

$$
\left(x_{1}, x_{2}, x_{3}\right) \cdot\left(y_{1}, y_{2}, y_{3}\right)=\left(x_{1}+y_{1}, x_{2}+y_{2}, x_{3}+y_{3}+\frac{1}{2}\left(x_{1} y_{2}-y_{1} x_{2}\right)\right) \text {. }
$$

Then

$$
H f(x)=\text { p.v. } \int_{-1}^{1} f\left(x \cdot \Gamma^{-1}(x)\right) \frac{d t}{t}
$$

and

$$
\mathscr{M} f(x)=\sup _{0<h \leq 1} \frac{1}{h} \int_{0}^{h}\left|f\left(x \cdot \Gamma^{-1}(t)\right)\right| d t
$$

where the multiplication is the Heisenberg group multiplication described above. We shall take $\Gamma(t)$ to be of the special form

$$
\Gamma(t)=(t, \gamma(t), t \gamma(t)), \quad \text { for } t>0 \text {. }
$$

This allows one to write $\Gamma(t)$ as

$$
\Gamma(t)=\delta(t) v \text { for } t>0
$$

where $v=(1,1,1)$ and

$$
\delta(t)=\operatorname{diag}(t, \gamma(t), t \gamma(t))
$$

are not only linear transformations on $\mathbb{R}^{3}$, but are also automorphisms of the Heisenberg group. It turns out that the appropriate curvature condition alluded to above will be satisfied exactly when $\gamma^{\prime \prime}(t)$ does not vanish to infinite order at $t=0$. So we shall be interested in the case $\gamma^{(j)}(0)=0$ for all $j$.

Our results are expressed in terms of the functional

$$
\lambda(t)=\frac{t \gamma^{\prime \prime}(t)}{\gamma^{\prime}(t)}
$$

Note that $\lambda(t)$ transforms well under scaling. That is if $\gamma_{a, b}(t)=a \gamma(b t)$,

$$
\lambda_{a, b}(t)=\frac{t \gamma_{a, b}^{\prime \prime}(t)}{\gamma_{a, b}^{\prime}(t)}=\lambda(b t) .
$$

Main Theorem. Suppose for $t>0$

$$
\Gamma(t)=(t, \gamma(t), t \gamma(t))
$$

with $\gamma(0)=\gamma^{\prime}(0)=0$, where $\gamma(t)$ is a convex curve in $C^{3}([0,1])$. Assume $\lambda(t)$ is decreasing and positive on $(0,1]$.

Furthermore assume

$$
\lim _{t \searrow 0} \frac{t \lambda^{\prime}(t)}{\lambda^{2}(t)} \log ^{+} \lambda(t)=0
$$

Then

$$
\|\mathscr{M} f\|_{L^{p}} \leq C(p)\|f\|_{L^{p}}, \quad 1<p \leq \infty,
$$

and if $\Gamma(t)$ is extended for negative $t$ to be an odd curve,

$$
\|H f\|_{L^{p}} \leq C(p)\|f\|_{L^{p}}, \quad 1<p<\infty .
$$


Remarks. The hypothesis of the main theorem seems rather technical, however it is straightforward to verify the hypothesis in examples such as

$$
\gamma(t)=\exp \left(-\frac{1}{t}\right), \text { or } \gamma(t)=\exp \left(-\exp \left(\frac{1}{t}\right)\right), \text { etc } \ldots
$$

We will actually prove the theorem for curves $\Gamma(t)=(t, \gamma(t), t \gamma(t))$ in a slightly more general setting. That is we will take the group law in $\mathbb{R}^{3}$ to be

$$
\left(x_{1}, x_{2}, x_{3}\right) \cdot\left(y_{1}, y_{2}, y_{3}\right)=\left(x_{1}+y_{1}, x_{2}+y_{2}, x_{3}+y_{3}+\beta\left(x_{1} y_{2}-y_{1} x_{2}\right)\right)
$$

where $x=\left(x_{1}, x_{2}, x_{3}\right), y=\left(y_{1}, y_{2}, y_{3}\right) \in \mathbb{R}^{3}$ and $\beta \in \mathbb{R}^{1}$ with $\beta \neq-1$, and consider $\gamma(x, t)=x \cdot \Gamma^{-1}(t)$ with the above multiplication. However for general $\beta$, we need to assume in addition that $\lambda(t)$ tends to infinity as $t$ tends to 0 . It will be evident that our proof does not directly apply to the case $\beta=-1$. Note that for $\beta=0$, we are in the setting of $(3), \gamma(x, t)=x-\Gamma(t)$; the Euclidean translation invariant case. One reason for studying these more general curves is that results for these curves easily imply results in $\mathbb{R}^{2}$. More specifically when $\beta=1$, we can use the diffeomorphism $\varphi(x, y, z)=(x, y, z+x y)$ on $\mathbb{R}^{3}$ to obtain $L^{p}$ estimates for the Hilbert transform and maximal function associated to the curves

$$
\gamma((x, y, z), t)=(x-t, y-\gamma(t), z-2 x \gamma(t)) .
$$

This in turn implies that the estimates (1) and (2) hold for the plane curves

$$
\gamma(x, y, t)=(x-t, y-x \gamma(t))
$$

when $\gamma(t)$ satisfies the hypothesis of the main theorem.

Since our basic operators do not commute with translations, the use of the Fourier transform does not seem to be a viable tool as in [CVWWA] or [CZ]. Instead we use ideas developed in [NSW], [C2], [RS] and [CVWWW]. Also we need to make use of a generalization of the space of homogeneous type as in [CW], developed in [CVWW].

\section{IDEA OF THE PROOF}

We will only give the proof of the estimate for the maximal function. The proof of the estimate for the Hilbert transform is similar. See [CVWWW], where the necessary modifications in the argument are explained. We set

$$
M_{k} f(x)=2^{k} \int_{2^{-k}}^{2^{-k+1}} f\left(x \cdot \Gamma^{-1}(t)\right) d t
$$

Let

$$
M f(x)=\sup _{k>0}\left|M_{k} f(x)\right| .
$$

It is not hard to see that $\mathscr{M} f(x) \leq C M f(x)$ for $f \geq 0$, and hence it suffices to show

$$
\|M f\|_{L^{p}} \leq C(p)\|f\|_{L^{p}}, \quad 1<p \leq \infty
$$


Let $A_{k}$ denote $\delta\left(2^{-k}\right)$, and $f_{k}(x)=f\left(A_{k} x\right)$. Then

$$
\begin{aligned}
M_{k} f(x) & =2^{k} \int_{2^{-k}}^{2^{-k+1}} f\left(x \cdot \Gamma^{-1}(t)\right) d t \\
& =\int_{1}^{2} f\left(x \cdot \Gamma^{-1}\left(2^{-k} t\right)\right) d t \\
& =\int_{1}^{2} f\left(A_{k}\left(A_{k}^{-1} x \cdot A_{k}^{-1} \Gamma^{-1}\left(2^{-k} t\right)\right)\right) d t \\
& =f_{k} * d \mu_{k}\left(A_{k}^{-1} x\right) .
\end{aligned}
$$

Here, for a test function $g$,

$$
\begin{aligned}
d \mu_{k}(g) & =\int_{1}^{2} g\left(A_{k}^{-1} \Gamma\left(2^{-k} t\right)\right) d t \\
& =\int_{1}^{2} g\left(\Gamma_{k}(t)\right) d t
\end{aligned}
$$

where

$$
\begin{aligned}
\Gamma_{k}(t) & =A_{k}^{-1} \Gamma\left(2^{-k} t\right)=\left(t, \frac{\gamma\left(2^{-k} t\right)}{\gamma\left(2^{-k}\right)}, t \frac{\gamma\left(2^{-k} t\right)}{\gamma\left(2^{-k}\right)}\right) \\
& =\left(t, \gamma_{k}(t), t \gamma_{k}(t)\right) .
\end{aligned}
$$

Also for a Radon measure $d \mu$,

$$
f * d \mu(x)=\int f\left(x \cdot y^{-1}\right) d \mu(y) .
$$

(More generally if $d \mu$ and $d \nu$ are two Radon measures,

$$
\left.d \nu * d \mu(f)=\iint f(z \cdot y) d \nu(z) d \mu(y) .\right)
$$

Let $d \mu_{k}^{*}$ be the measure defined on test functions $g$ by

$$
d \mu_{k}^{*}(g)=\int_{1}^{2} g\left(\Gamma_{k}^{-1}(t)\right) d t .
$$

The operation $f \rightarrow f * d \mu_{k}^{*}$ is the adjoint of $f \rightarrow f * d \mu_{k}$.

The essence of the proof of the main theorem is to show that

$$
d \mu_{k} * d \mu_{k}^{*} * d \mu_{k} * d \mu_{k}^{*}
$$

has an $L^{1}$ density $\rho_{k}$ with a certain amount of $L^{1}$ smoothness. Note that

$$
M_{k}^{*} M_{k} M_{k}^{*} M_{k} f(x)=f_{k} * d \mu_{k} * d \mu_{k}^{*} * d \mu_{k} * d \mu_{k}^{*}\left(A_{k}^{-1} x\right) .
$$

In fact we shall show that $d \mu_{k} * d \mu_{k}^{*} * d \mu_{k} * d \mu_{k}^{*}$ has a density $\rho_{k}$ and that

$$
\rho_{k}=\rho_{k, 1}+\rho_{k, 2}
$$

where for some $\epsilon>0$

$$
\int\left|\rho_{k, 1}(x \cdot y)-\rho_{k, 1}(x)\right| d x \leq C h^{\epsilon}
$$


whenever $\|y\|<h$, and

$$
\int\left|\rho_{k, 2}(y \cdot x)-\rho_{k, 2}(x)\right| d x \leq C h^{\epsilon}
$$

whenever $\|y\|<h$. Once we prove (1.2) and (1.3) we can follow the general ideas of [C2] or [RS] to complete the proof of the main theorem.

Now if $f$ is a test function,

$$
\begin{aligned}
d \mu_{k} * d \mu_{k}^{*} * d \mu_{k} * d \mu_{k}^{*}(f) & \\
& =\int_{1}^{2} \int_{1}^{2} \int_{1}^{2} \int_{1}^{2} f\left(\Gamma_{k}(s) \cdot \Gamma_{k}^{-1}(t) \cdot \Gamma_{k}(u) \cdot \Gamma_{k}^{-1}(w)\right) d s d t d u d w .
\end{aligned}
$$

As in [C2] and [RS], we will divide the region of integration into a number of parts. In each part we will fix one variable and make a change of variables in the other three. Suppose for example we are in a region where we fix $t$. Consider the mapping

$$
\varphi_{t}(s, u, w)=\Gamma_{k}(s) \cdot \Gamma_{k}^{-1}(t) \cdot \Gamma_{k}(u) \cdot \Gamma_{k}^{-1}(w)
$$

which maps $\mathbb{R}^{3}$ into the Heisenberg group, and make the change of variables

$$
x=\varphi_{t}(s, u, w)
$$

in the integral. The new difficulty that arises is that we have no uniform control over the size of the derivatives of $\varphi_{t}$. So for example it becomes difficult to estimate the size of sets on which the transformation (1.4) is one to one. Also formally, one finds that the density $\rho(x)$ is $1 /\left|J_{\varphi_{t}}\left(\varphi_{t}^{-1}(x)\right)\right|$ where $J_{\varphi_{t}}$ denotes the Jacobian of $\varphi_{t}$. Thus to estimate the smoothness of $\rho(x)$, one would like to have estimates on the derivatives of $\varphi_{t}$ which are not available.

Our basic idea is to divide the cube

$$
Q=\left\{(s, t, u, w) \in \mathbb{R}^{4} \mid 1 \leq s, t, u, w \leq 2\right\}
$$

into two parts. One part will have small area. On this part we will largely follow the argument of [RS] and use the fact that the area of this part is small to overcome the lack of uniformity in the control of the size of the derivatives of $\varphi_{t}$. On the other part we make a suitable approximation to the Jacobian of $\varphi_{t}, J_{\varphi_{1}}$, so that we may directly calculate that the argument of [CVWWW] applies.

\section{THE MAIN ESTIMATE-SOME PRELIMINARY SPLITTING}

We shall assume $\lambda(t)$ tends to infinity as $t$ tends to 0 . If $\lambda(t)$ stays bounded the proof is much easier for certain $\beta$, including $\beta=1 / 2$. A monotonicity argument is needed which is not valid for general $\beta$. Furthermore since we are only concerned with what happens for $t$ small, we shall assume $\lambda(t)$ is arbitrarily large. We proceed to the proof of (1.1), (1.2) and (1.3) above. 
For a test function $f$

$$
\begin{aligned}
d \mu_{k} * d \mu_{k}^{*} * d \mu_{k} * d \mu_{k}^{*}(f) & \\
= & \int_{Q} f\left(\Gamma_{k}(s) \cdot \Gamma_{k}^{-1}(t) \cdot \Gamma_{k}(u) \cdot \Gamma_{k}^{-1}(w)\right) d s d t d u d w \\
= & \int_{Q_{1}} f(\varphi(s, t, u, w)) d s d t d u d w \\
& \quad+\int_{Q_{2}} f(\varphi(s, t, u, w)) d s d t d u d w \\
= & \Theta_{1}(f)+\Theta_{2}(f)
\end{aligned}
$$

Here

$$
\begin{aligned}
Q & =\left\{(s, t, u, w) \in \mathbb{R}^{4} \mid 1 \leq s, t, u, w \leq 2\right\}, \\
Q_{1} & =\{(s, t, u, w) \in Q \mid \max \{s, t, u, w\}=\max \{s, t\}\}, \\
Q_{2} & =\{(s, t, u, w) \in Q \mid \max \{s, t, u, w\}=\max \{u, w\}\},
\end{aligned}
$$

and

$$
\varphi(s, t, u, w)=\Gamma_{k}(s) \cdot \Gamma_{k}^{-1}(t) \cdot \Gamma_{k}(u) \cdot \Gamma_{k}(w)^{-1} .
$$

We shall show that $\Theta_{1}$ has a density $\rho_{k, 1}$ satisfying (1.2). Note that

$$
\Theta_{2}(f)=\int_{Q_{1}} f\left(\varphi^{-1}(s, t, u, w)\right) d s d t d u d w
$$

and so

$$
\begin{aligned}
\boldsymbol{\Theta}_{2}(f) & =\int f\left(x^{-1}\right) \rho_{k, 1}(x) d x \\
& =\int f(x) \rho_{k, 1}\left(x^{-1}\right) d x .
\end{aligned}
$$

Thus $\rho_{k, 2}(x)=\rho_{k, 1}\left(x^{-1}\right)$ and so (1.3) follows from (1.2). Therefore we shall only prove (1.2).

We now divide $Q_{1}$ into four parts, $Q_{1}=R_{1} \cup R_{2} \cup R_{3} \cup R_{4}$,

$$
\begin{array}{ll}
R_{1}=\left\{(s, t, u, w) \in Q_{1} \mid \max \{s, t\}=s,\right. & u<w\}, \\
R_{2}=\left\{(s, t, u, w) \in Q_{1} \mid \max \{s, t\}=s,\right. & w<u\}, \\
R_{3}=\left\{(s, t, u, w) \in Q_{1} \mid \max \{s, t\}=t,\right. & u<w\},
\end{array}
$$

and

$$
R_{4}=\left\{(s, t, u, w) \in Q_{1} \mid \max \{s, t\}=t, w<u\right\} .
$$

We will discuss in detail the contribution to $\Theta_{1}$ from integrating over $R_{1}$. The contributions from $R_{2}, R_{3}$ and $R_{4}$ are treated similarly. Roughly the difference is that in treating $R_{1}$ and $R_{2}$ we fix $t, 1 \leq t \leq 2$, and make a change of variables $x=\varphi_{t}(s, u, w)=\varphi(s, t, u, w)$ from $\mathbb{R}^{3}$ to $\mathbb{R}^{3}$. In $R_{3}$ and $R_{4}$ we fix $s$ and consider the change of variables $x=\varphi_{s}(t, u, w)=\varphi(s, t, u, w)$. In $R_{1}$ and $R_{2}$ we make different approximations to the Jacobian of $\varphi_{t}$. Similarly we use different approximations to the Jacobian of $\varphi_{s}$ in $R_{3}$ and $R_{4}$. It will be clear what modifications to make in the Jacobians. 
We must now analyze

$$
\begin{aligned}
\int_{R_{1}} f(\varphi(s, t, u, w)) d s d t d u d w \\
\quad=\int_{1}^{2}\left\{\int_{R(t)} f\left(\varphi_{t}(s, u, w)\right) d s d u d w\right\} d t
\end{aligned}
$$

where

$$
R(t)=\left\{(s, u, w) \in \mathbb{R}^{3} \mid(s, t, u, w) \in R_{1}\right\}
$$

Let

$$
\Theta^{t}(f)=\int_{R(t)} f\left(\varphi_{t}(s, u, w)\right) d s d u d w
$$

Clearly it suffices to show that $\Theta^{t}$ has an $L^{1}$ density $\rho_{t}$ such that 1.2$)$ holds uniformly in $t$.

\section{The Balls}

We begin by dividing $R(t)$ into three regions. We set

$$
P_{t}(s, u, w)=s-w+\beta(w-s+2 t-2 u) .
$$

The significance of $P_{t}$ is the set where our approximation to $J_{\varphi_{t}}$ vanishes is precisely when $P_{t}=0$. We should remark here that in the region where the variable $t$ is the largest (and thus we fix $s$ ) and $w<u$, the above polynomial is

$$
P_{s}(t, u, w)=t-u+\beta(t-u)
$$

which vanishes identically when $\beta=-1$. Therefore our approximation to $J_{\varphi_{s}}$ will not hold in this region and so a different argument is needed in this case.

Now let $C$ be a large constant to be determined later. Set

$$
\begin{aligned}
& \Omega_{k}^{1}=\left\{(s, u, w) \in R(t) \mid w-u \geq C \frac{\log \lambda\left(2^{-k} u\right)}{\lambda\left(2^{-k} u\right)},\right. \\
& \left.\quad \text { and }\left|P_{t}(s, u, w)\right| \geq C \frac{1}{\lambda\left(2^{-k} s\right)}\right\},
\end{aligned}
$$

$$
\begin{aligned}
& \Omega_{k}^{2}=\left\{(s, u, w) \in R(t) \mid w-u \leq C \frac{\log \lambda\left(2^{-k} u\right)}{\lambda\left(2^{-k} u\right)},\right. \\
&\text { and } \left.J_{\varphi_{t}}(s, u, w) \neq 0\right\},
\end{aligned}
$$

and

$$
\begin{aligned}
\Omega_{k}^{3}=\left\{(s, u, w) \in R(t) \mid w-u>C \frac{\log \lambda\left(2^{-k} u\right)}{\lambda\left(2^{-k} u\right)}\right. \\
\left.\quad\left|P_{t}(s, u, w)\right| \leq \frac{c}{\lambda\left(2^{-k} s\right)} \text { and } J_{\varphi_{t}}(s, u, w) \neq 0\right\} .
\end{aligned}
$$


Let $X$ denote the hypersurface

$$
w-u=C \frac{\log \lambda\left(2^{-k} u\right)}{\lambda\left(2^{-k} u\right)},
$$

and $Y$ denote the hypersurface

$$
\left|P_{t}(s, u, w)\right|=C \frac{1}{\lambda\left(2^{-k} s\right)} .
$$

Once we show the measure of the zero set of $J_{\varphi_{t}}$ is zero, we shall have an essential decomposition of $R(t)$,

$$
R(t)=\Omega_{k}^{1} \cup \Omega_{k}^{2} \cup \Omega_{k}^{3} .
$$

Our first type of ball is derived from a Whitney decomposition of $\Omega_{k}^{1}$. As in Stein, [S], we find cubes $Q_{\ell, n}$ such that

$$
\Omega_{k}^{1}=\cup Q_{\ell, n},
$$

$$
\operatorname{diameter}\left(Q_{\ell, n}\right)=\epsilon_{0} 2^{-\ell}
$$

for a suitable $\epsilon_{0}>0$,

$$
2^{-\ell-1} \leq \operatorname{dist}\left(\text { center of } Q_{\ell, n}, \text { boundary of } \Omega_{k}^{1}\right) \leq 2^{-\ell} .
$$

There is a constant $C$ so that no point is in more than $C$ of the $Q_{\ell n}^{*}$. Here $Q_{\ell n}^{*}$ is the cube with the same center as $Q_{\ell n}$ and having a side length twice as large. that

Since $\Omega_{k}^{1}$ is bounded by a finite number of smooth hypersurfaces, it is clear

(3.9) for a given $\ell$, the number of cubes $Q_{\ell n}$ is at most $C 2^{2 \ell}$.

Our second type of ball will be contained in $\Omega_{k}^{2}$. For $p=(s, u, w) \in \Omega_{k}^{2}$ and $\epsilon>0$ small, set

$$
\begin{aligned}
& r_{1}(p)=\epsilon \frac{\left|J_{\varphi_{t}}(p)\right|}{\gamma_{k}^{\prime \prime}(s) \gamma_{k}^{\prime}(w)} \Delta(p), \\
& r_{2}(p)=\epsilon \frac{\left|J_{\varphi_{t}}(p)\right|}{\gamma_{k}^{\prime \prime}(w) \gamma_{k}^{\prime}(s)} \Delta(p),
\end{aligned}
$$

and

$$
r_{3}(p)=\epsilon \frac{\left|J_{\varphi_{t}}(p)\right|}{\gamma_{k}^{\prime \prime}(w) \gamma_{k}^{\prime}(s)} \Delta(p)
$$


where

$$
\Delta(p)=(s-t)(w-u) \operatorname{dist}(p, X) \operatorname{dist}(p, Y) .
$$

We then form balls

$$
\begin{aligned}
& D\left(p_{0}\right)=\{(s, u, w) \mid s_{0}-r_{1}\left(p_{0}\right) \leq s \leq s_{0}+r_{1}\left(p_{0}\right), \\
& w_{0}-r_{2}\left(p_{0}\right) \leq w \leq w_{0}+r_{2}\left(p_{0}\right), \\
&\left.u_{0}-r_{3}\left(p_{0}\right) \leq u \leq u_{0}+r_{3}\left(p_{0}\right)\right\},
\end{aligned}
$$

and the corresponding doubles

$$
\begin{array}{r}
D^{*}\left(p_{0}\right)=\left\{(s, u, w) \mid s_{0}-2 r_{1}\left(p_{0}\right) \leq s \leq s_{0}+2 r_{1}\left(p_{0}\right),\right. \\
w_{0}-2 r_{2}\left(p_{0}\right) \leq w \leq w_{0}+2 r_{2}\left(p_{0}\right), \\
\left.u_{0}-2 r_{3}\left(p_{0}\right) \leq u \leq u_{0}+2 r_{3}\left(p_{0}\right)\right\} .
\end{array}
$$

We define a third type of ball for $\Omega_{k}^{3}$. For $p \in \Omega_{k}^{3}$, we set

$$
\begin{aligned}
& \tilde{r}_{1}(p)=\epsilon \frac{\left|J_{\varphi_{t}}(p)\right|}{\gamma_{k}^{\prime \prime}(s) \gamma_{k}^{\prime}(w)} \Delta(p), \\
& \tilde{r}_{2}(p)=\epsilon \frac{\left|J_{\varphi_{t}}(p)\right|}{\gamma_{k}^{\prime \prime}(w) \gamma_{k}^{\prime}(s)} \Delta(p),
\end{aligned}
$$

and

$$
\tilde{r}_{3}(p)=\epsilon \frac{\left|J_{\varphi_{t}}(p)\right|}{\gamma_{k}^{\prime}(s) \gamma_{k}^{\prime}(s)} \Delta(p) .
$$

If $p_{0}=\left(s_{0}, u_{0}, w_{0}\right) \in \Omega_{k}^{3}$, set

$$
\begin{array}{r}
G\left(p_{0}\right)=\left\{(s, u, w) \mid s_{0}-\tilde{r}_{1}\left(p_{0}\right) \leq s \leq s_{0}+\tilde{r}_{1}\left(p_{0}\right)\right. \\
w_{0}-\tilde{r}_{2}\left(p_{0}\right) \leq w \leq w_{0}+\tilde{r}_{2}\left(p_{0}\right), \\
\left.u_{0}-\tilde{r}_{3}\left(p_{0}\right) \leq u \leq u_{0}+\tilde{r}_{3}\left(p_{0}\right)\right\}
\end{array}
$$

and the corresponding doubles

$$
\begin{array}{r}
G^{*}\left(p_{0}\right)=\left\{(s, u, w) \mid s_{0}-2 \tilde{r}_{1}\left(p_{0}\right) \leq s \leq s_{0}+2 \tilde{r}_{1}\left(p_{0}\right)\right. \\
w_{0}-2 \tilde{r}_{2}\left(p_{0}\right) \leq w \leq w_{0}+2 \tilde{r}_{2}\left(p_{0}\right), \\
\left.u_{0}-2 \tilde{r}_{3}\left(p_{0}\right) \leq u \leq u_{0}+2 \tilde{r}_{3}\left(p_{0}\right)\right\} .
\end{array}
$$

If $p_{0}=\left(s_{0}, u_{0}, w_{0}\right) \in Q_{\ell n} \subset \Omega_{k}^{1}$, let

$$
B\left(p_{0}\right)=Q_{\ell n}^{*} \text {. }
$$

If $p_{0} \in \Omega_{k}^{2}$, let

$$
B\left(p_{0}\right)=D^{*}\left(p_{0}\right)
$$

and finally if $p_{0} \in \Omega_{k}^{3}$, let

$$
B\left(p_{0}\right)=G^{*}\left(p_{0}\right)
$$

So $\left\{B\left(p_{0}\right)\right\}$ covers all of $R(t)$ except perhaps for a set of measure zero. We shall prove that we can find a good partition of unity of $R(t)$ subordinate to a 
subcollection of the balls $\{B(p)\}$, and that $\varphi_{t}$ is one to one on each ball $B(p)$. Note that if $\varphi_{t}$ is one to one on $B(p)$ and $\psi$ is supported in $B(p)$,

$$
\begin{aligned}
& \int \psi(s, u, w) f\left(\varphi_{t}(s, u, w)\right) d s d u d w \\
& \quad=\int \psi\left(\varphi_{t}^{-1}(x)\right) \frac{1}{\left|J_{\varphi_{t}}\left(\varphi_{t}^{-1}(x)\right)\right|} f(x) d x
\end{aligned}
$$

Thus we will have a contribution of

$$
K(x)=\psi\left(\varphi_{t}^{-1}(x)\right) \frac{1}{\left|J_{\varphi_{t}}\left(\varphi_{t}^{-1}(x)\right)\right|}
$$

to the density $\rho_{t}$. In order to prove (1.2) we will have to estimate $L_{j} K(x)$, where $L_{1}=\frac{\partial}{\partial x_{1}}-\beta x_{2} \frac{\partial}{\partial x_{3}}, L_{\hat{2}}=\frac{\partial}{\partial x_{2}}+\beta x_{1} \frac{\partial}{\partial x_{3}}$ and $L_{3}=\frac{\partial}{\partial x_{3}}$ are left invariant vector fields on the Heisenberg group. By left invariance we see that

$$
L_{j} K(x)=\left.\frac{\partial}{\partial y_{j}}(K(x \cdot y))\right|_{y=0}, \quad j=1,2,3 .
$$

Since we know explicitly what $K(x)$ is in the $(s, u, w)$ coordinates, it will facilitate matters to express the above differential operators in the $(s, u, w)$ coordinates. To do this let $\left(s_{0}, u_{0}, w_{0}\right) \in B(p)$ be a point in the support of $\psi$ and consider the mapping

$$
f(s, u, w)=\varphi_{t}^{-1}\left(s_{0}, u_{0}, w_{0}\right) \cdot \varphi_{t}(s, u, w)
$$

which maps diffeomorphically a neighborhood of $\left(s_{0}, u_{0}, w_{0}\right)$ onto a neighborhood of the identity of the Heisenberg group. Therefore the differential of $f^{-1}, d\left(f^{-1}\right)$, maps the tangent space at the identity of the Heisenberg group to the tangent space at $\left(s_{0}, u_{0}, w_{0}\right)$ of $\mathbb{R}^{3}$. We are interested in the image of the tangent vectors $\left.\frac{\partial}{\partial y_{j}}\right|_{y=0}, j=1,2,3$, under the mapping $d\left(f^{-1}\right)$. By the chain rule we can express $d\left(f^{-1}\right)\left(\left.\frac{\partial}{\partial y_{j}}\right|_{y=0}\right)$ as

$$
\left\langle\left(f^{-1}\right)^{\prime} e_{j},\left.\left(\frac{\partial}{\partial s}, \frac{\partial}{\partial u}, \frac{\partial}{\partial w}\right)\right|_{s_{0}, u_{0}, w_{0}}\right\rangle
$$

where $\left(f^{-1}\right)^{\prime}$ denotes the Jacobian matrix of $f^{-1}, e_{1}=(1,0,0), e_{2}=$ $(0,1,0)$ and $e_{3}=(0,0,1)$. Note that

$$
d(f)\left(s_{0}, u_{0}, w_{0}\right)=d L_{x^{-1}} \circ d\left(\varphi_{t}\right)\left(s_{0}, u_{0}, w_{0}\right)
$$

where $L_{x^{-1}}$ is the operation of left multiplication by $x^{-1}=\varphi_{t}^{-1}\left(s_{0}, u_{0}, w_{0}\right)$ on the Heisenberg group. Therefore the Jacobian matrix for $f$ at $\left(s_{0}, u_{0}, w_{0}\right)$ is

$$
\mathscr{G}\left(s_{0}, u_{0}, w_{0}\right)=\left(\begin{array}{ccc}
1 & 0 & 0 \\
0 & 1 & 0 \\
\beta x_{2} & -\beta x_{1} & 1
\end{array}\right) \varphi_{t}^{\prime}\left(s_{0}, u_{0}, w_{0}\right)
$$


Here $\left(x_{1}, x_{2}, x_{3}\right)$ are the coordinates for the point $x=\varphi_{t}\left(s_{0}, u_{0}, w_{0}\right)$. Finally we see that $\left(f^{-1}\right)^{\prime}=\mathscr{G}^{-1}$ and so (3.23) may be expressed as

$$
d\left(f^{-1}\right)\left(\left.\frac{\partial}{\partial y_{j}}\right|_{y=0}\right)=\left\langle\left.\left(\mathscr{G}^{-1}\right)^{*}\left(s_{0}, u_{0}, w_{0}\right)\left(\frac{\partial}{\partial s}, \frac{\partial}{\partial u}, \frac{\partial}{\partial w}\right)\right|_{\left(s_{0}, u_{0}, w_{0}\right)}, e_{j}\right\rangle .
$$

Let us write

$$
\left(\mathscr{G}^{-1}\right)^{*}=\frac{1}{\operatorname{det} \mathscr{G}}\left(g_{i j}\right)=\frac{1}{J_{\varphi_{t}}} \cdot\left(g_{i j}\right)
$$

where $g_{i j}$ is the $(i, j)$ cofactor of the matrix $\mathscr{G}$. Thus we will need estimates on $\frac{g_{i j}}{\operatorname{det} \mathscr{G}}$ and the corresponding derivatives. In order to obtain these estimates we first need a few lemmas.

\section{Some Lemmas}

In this section we derive some consequences of the hypothesis on $\lambda$.

Lemma 4.1. Let $C_{0}$ and $\delta$ be positive. If $1 \leq w \leq 2$ and

$$
0 \leq w-u \leq C_{0} \frac{\log \lambda\left(2^{-k} u\right)}{\lambda\left(2^{-k} u\right)}
$$

then

$$
\lambda\left(2^{-k} u\right) \leq e^{2 \delta C_{0}} \lambda\left(2^{-k} w\right)
$$

Remark. Recall that we are assuming that $\lambda$ is arbitrarily large and therefore we may assume that $k$ is arbitrarily large in what follows.

Proof.

$$
\log \frac{\lambda\left(2^{-k} u\right)}{\lambda\left(2^{-k} w\right)}=-\int_{u}^{w} \frac{2^{-k} r \lambda^{\prime}\left(2^{-k} r\right)}{\lambda\left(2^{-k} r\right)} \frac{d r}{r}
$$

From (7)

$$
\left|\frac{2^{-k} r \lambda^{\prime}\left(2^{-k} r\right)}{\lambda\left(2^{-k} r\right)}\right| \leq \delta \frac{\lambda\left(2^{-k} r\right)}{\log \lambda\left(2^{-k} r\right)}
$$

for $k$ large enough and $r \leq 2$. Thus since $u \geq \frac{1}{2}$ for $k$ large,

$$
\begin{aligned}
\log \frac{\lambda\left(2^{-k} u\right)}{\lambda\left(2^{-k} w\right)} & \leq 2 \delta \int_{u}^{w} \frac{\lambda\left(2^{-k} r\right)}{\log \lambda\left(2^{-k} r\right)} d r \\
& \leq 2 \delta \frac{\lambda\left(2^{-k} u\right)}{\log \lambda\left(2^{-k} u\right)}(w-u) \leq 2 C_{0} \delta .
\end{aligned}
$$

The lemma follows by exponentiating the last inequality.

Lemma 4.2. Let $1 \leq w \leq 2$, and assume $w-u \geq C_{0} \frac{\log \lambda\left(2^{-k} u\right)}{\lambda\left(2^{-k} u\right)}$. Then for $k$ large enough

$$
\frac{\gamma_{k}^{\prime}(w)}{\gamma_{k}^{\prime}(u)} \geq\left[\lambda\left(2^{-k} u\right)\right]^{C_{0} / 4}
$$


Proof. Since $\gamma_{k}^{\prime}$ is increasing and $\lambda$ is decreasing, we may assume $w-u=$ $C_{0} \frac{\log \lambda\left(2^{-k} u\right)}{\lambda\left(2^{-k} u\right)}$. Then

$$
\begin{aligned}
\log \frac{\gamma_{k}^{\prime}(w)}{\gamma_{k}^{\prime}(u)} & =\int_{u}^{w} \lambda\left(2^{-k} r\right) \frac{d r}{r} \geq \frac{1}{2} \lambda\left(2^{-k} w\right)(w-u) \\
& \geq \frac{1}{4} \lambda\left(2^{-k} u\right)(w-u)=\frac{C_{0}}{4} \log \lambda\left(2^{-k} u\right)
\end{aligned}
$$

by Lemma 4.1 if $k$ is large enough. The result follows now by exponentiating. Lemma 4.3. Suppose $1 \leq w \leq 2$. Then

$$
\frac{\gamma_{k}^{\prime}(w)}{\gamma_{k}(w)} \geq \frac{1}{2} \lambda\left(2^{-k} w\right)
$$

Proof.

$$
\frac{\gamma_{k}(w)}{\gamma_{i}^{\prime}(w)}=\int_{0}^{w} \frac{\gamma_{k}^{\prime}(r)}{\gamma_{k}^{\prime}(w)} d r
$$

But

$$
\log \frac{\gamma_{k}^{\prime}(w)}{\gamma_{k}^{\prime}(r)}=\int_{r}^{w} \lambda\left(2^{-k} \sigma\right) \frac{d \sigma}{\sigma} \geq \frac{1}{2} \lambda\left(2^{-k} w\right)(w-r),
$$

which implies

$$
\frac{\gamma_{k}^{\prime}(r)}{\gamma_{k}^{\prime}(w)} \leq e^{-\frac{1}{2} \lambda\left(2^{-k} w\right)(w-r)}
$$

So

$$
\begin{aligned}
\frac{\gamma_{k}(w)}{\gamma_{k}^{\prime}(w)} & \leq \int_{0}^{w} e^{-\frac{1}{2}(w-r) \lambda\left(2^{-k} w\right)} d r \\
& \leq \frac{2}{\lambda\left(2^{-k} w\right)} \int_{0}^{\infty} e^{-t} d t \\
& =\frac{2}{\lambda\left(2^{-k} w\right)}
\end{aligned}
$$

which proves the lemma.

Lemma 4.4. There are positive constants $C_{1}$ and $C_{2}$ so that if $1 \leq t \leq 2$ and

$$
t-\frac{t}{\lambda\left(2^{-k} t\right)} \leq r, \quad s \leq t+\frac{t}{\lambda\left(2^{-k} t\right)},
$$

then

$$
\begin{aligned}
& C_{1} \leq \frac{\lambda\left(2^{-k} r\right)}{\lambda\left(2^{-k} s\right)} \leq C_{2}, \\
& C_{1} \leq \frac{\gamma\left(2^{-k} r\right)}{\gamma\left(2^{-k} s\right)} \leq C_{2}, \\
& C_{1} \leq \frac{\gamma_{k}^{\prime}\left(2^{-k} r\right)}{\gamma_{k}^{\prime}\left(2^{-k} s\right)} \leq C_{2},
\end{aligned}
$$


and

$$
C_{1} \leq \frac{\gamma_{k}^{\prime \prime}\left(2^{-k} r\right)}{\gamma_{k}^{\prime \prime}\left(2^{-k} s\right)} \leq C_{2} .
$$

Proof. To prove (4.1) note that it suffices to prove

$$
\frac{\lambda\left(t-\frac{t}{\lambda(t)}\right)}{\lambda\left(t+\frac{t}{\lambda(t)}\right)} \leq C
$$

for $t$ small. First by (7),

$$
\begin{aligned}
\log \frac{\lambda(t)}{\lambda\left(t+\frac{t}{\lambda(t)}\right)} & =-\int_{t}^{t+\frac{t}{\lambda(t)}} \frac{\lambda^{\prime}(\sigma)}{\lambda(\sigma)} d \sigma \\
& \leq \delta \int_{t}^{t+\frac{t}{\lambda(t)}} \frac{\lambda(\sigma)}{\sigma} d \sigma
\end{aligned}
$$

where $\delta>0$ is small if $t$ is small. Since $\frac{\lambda(\sigma)}{\sigma}$ is decreasing, we may estimate the last integral by 1 and so

$$
\frac{\lambda(t)}{\lambda\left(t+\frac{t}{\lambda(t)}\right)} \leq e^{\delta}
$$

if $t$ is small. Therefore it suffices to prove

$$
\frac{\lambda\left(t-\frac{t}{\lambda(t)}\right)}{\lambda(t)} \leq C \text {. }
$$

Set $s=\lambda(t)$ and $s^{\prime}=\lambda\left(t-\frac{t}{\lambda(t)}\right)=\lambda\left(\lambda^{-1}(s)\left(1-\frac{1}{s}\right)\right)$. We will show that $s^{\prime} \leq 2 s$ which in turn implies (4.1). The estimate $s^{\prime} \leq 2 s$ will follow from the estimate $\lambda^{-1}(2 s) \leq \lambda^{-1}\left(s^{\prime}\right)$ or

$$
\lambda^{-1}(2 s) \leq \lambda^{-1}(s)\left(1-\frac{1}{s}\right)
$$

But from (7),

$$
\begin{aligned}
\log \frac{\lambda^{-1}(s)}{\lambda^{-1}(2 s)} & =-\int_{s}^{2 s} \frac{1}{\lambda^{\prime}\left(\lambda^{-1}(\sigma)\right)} \frac{1}{\lambda^{-1}(\sigma)} d \sigma \\
& \geq \frac{1}{\delta} \int_{s}^{2 s} \frac{1}{\sigma^{2}} d \sigma=\frac{1}{2 \delta s}
\end{aligned}
$$

where $\delta>0$ is small if $t$ is small (hence $s$ is large). Exponentiating this inequality gives us

$$
\frac{\lambda^{-1}(2 s)}{\lambda^{-1}(s)} \leq e^{-\frac{1}{2 \delta s}} \leq 1-\frac{1}{4 \delta s} \leq 1-\frac{1}{s}
$$

if $\delta<\frac{1}{4}$ and $s$ is large enough. This establishes (4.1). 
If $r>s$,

$$
\begin{aligned}
\log \frac{\gamma^{\prime}\left(2^{-k} r\right)}{\gamma^{\prime}\left(2^{-k} s\right)} & =\int_{s}^{r} \frac{2^{-k} u \gamma^{\prime \prime}\left(2^{-k} u\right)}{\gamma^{\prime}\left(2^{-k} u\right)} \frac{d u}{u} \\
& =\int_{s}^{r} \lambda\left(2^{-k} u\right) \frac{d u}{u} \leq 2(r-s) \lambda\left(2^{-k} s\right) \\
& \leq 8 \frac{\lambda\left(2^{-k} s\right)}{\lambda\left(2^{-k} t\right)} \leq C
\end{aligned}
$$

by (4.1) which implies (4.3). Next (4.4) follows from (4.1), (4.3) and the definition of $\lambda$. Finally to prove (4.2) for $r>s$ write

$$
\gamma\left(2^{-k} r\right)-\gamma\left(2^{-k} s\right)=\int_{s}^{r} 2^{-k} \gamma^{\prime}\left(2^{-k} u\right) d u \leq 2^{-k} \gamma^{\prime}\left(2^{-k} r\right)(r-s) .
$$

Dividing by $\gamma\left(2^{-k} s\right)$ yields

$$
\frac{\gamma\left(2^{-k} r\right)}{\gamma\left(2^{-k} s\right)} \leq 1+C \frac{\gamma^{\prime}\left(2^{-k} r\right)}{\gamma^{\prime}\left(2^{-k} s\right)} .
$$

Then (4.2) follows from (4.3).

Lemma 4.5. If $1 \leq u<s \leq 2$,

$$
\gamma_{k}^{\prime}(s)-\gamma_{k}^{\prime}(u) \geq \epsilon \gamma_{k}^{\prime}(s)(s-u)
$$

for some positive $\epsilon$.

Proof. We may assume $\gamma_{k}^{\prime}(u) \geq \frac{1}{2} \gamma_{k}^{\prime}(s)$. Then

$$
\begin{aligned}
\gamma_{k}^{\prime}(s)-\gamma_{k}^{\prime}(u) & =\int_{u}^{s} \gamma_{k}^{\prime \prime}(r) d r \\
& \geq \frac{1}{2} \int_{u}^{s} \gamma_{k}^{\prime}(r) \lambda\left(2^{-k} r\right) d r \geq \epsilon \gamma_{k}^{\prime}(u)(s-u)
\end{aligned}
$$

since we are assuming that $\lambda$ is bounded below. The proof is now complete since $\gamma_{k}^{\prime}(u) \geq \frac{1}{2} \gamma_{k}^{\prime}(s)$.

\section{SOME ESTIMATES FOR THE JACOBian OF $\varphi_{t}$,} AND APPLICATIONS TO $r_{1}, r_{2}, r_{3}, \tilde{r}_{1}, \tilde{r}_{2}$, AND $\tilde{r}_{3}$

If $a=\left(a_{1}, a_{2}, a_{3}\right)$ and $b=\left(b_{1}, b_{2}, b_{3}\right)$ are two vectors in $\mathbb{R}^{3}$, then we define $[a, b]$ as the vector

$$
[a, b]=\left(0,0, a_{1} b_{2}-b_{1} a_{2}\right) .
$$

With this notation

$$
\begin{aligned}
\varphi_{t}(s, u, w)= & \Gamma_{k}(s)-\Gamma_{k}(t)+\Gamma_{k}(u)-\Gamma_{k}(w) \\
& +\beta\left[\Gamma_{k}(s),-\Gamma_{k}(t)+\Gamma_{k}(u)-\Gamma_{k}(w)\right] \\
& +\beta\left[-\Gamma_{k}(t), \Gamma_{k}(u)-\Gamma_{k}(w)\right]+\beta\left[\Gamma_{k}(u),-\Gamma_{k}(w)\right]
\end{aligned}
$$


Recall from the end of Section 3 that $J_{\varphi_{t}}(s, u, w)=\operatorname{det} \mathscr{G}(s, u, w)$ where

$$
\mathscr{G}(s, u, w)=\left(\begin{array}{ccc}
1 & 0 & 0 \\
0 & 1 & 0 \\
\beta x_{2} & -\beta x_{1} & 1
\end{array}\right) \varphi_{t}^{\prime}(s, u, w)
$$

Here $x=\left(x_{1}, x_{2}, x_{3}\right)=\varphi_{t}(s, u, w)$. A calculation shows

$$
\mathscr{G}(s, u, w)=
$$

$$
\left(\begin{array}{ccc}
\Gamma_{k}^{\prime}(s) & \Gamma_{k}^{\prime}(u) & -\Gamma_{k}^{\prime}(w) \\
+\beta\left[\Gamma_{k}^{\prime}(s), \Gamma_{k}(s)\right] & +\beta\left[\Gamma_{k}^{\prime}(u), \Gamma_{k}(u)\right] & +\beta\left[\Gamma_{k}^{\prime}(w), \Gamma_{k}(w)\right] \\
+2 \beta\left[\Gamma_{k}^{\prime}(s),-\Gamma_{k}(t)+\Gamma_{k}(u)-\Gamma_{k}(w)\right] & +2 \beta\left[\Gamma_{k}^{\prime}(u),-\Gamma_{k}(w)\right] &
\end{array}\right)
$$

and

$$
\mathscr{G}(s, u, w)=\left(\begin{array}{ccc}
1 & 1 & -1 \\
\gamma_{k}^{\prime}(s) & \gamma_{k}^{\prime}(u) & -\gamma_{k}^{\prime}(w) \\
A & C & -D
\end{array}\right)
$$

where

$$
\begin{aligned}
A= & \left(s \gamma_{k}(s)\right)^{\prime}-\beta\left(s \gamma_{k}^{\prime}(s)-\gamma_{k}(s)\right)+2 \beta\left(-\gamma_{k}(t)+\gamma_{k}(u)-\gamma_{k}(w)\right) \\
& -2 \beta(-t+u-w) \gamma_{k}^{\prime}(s), \\
C= & \left(u \gamma_{k}(u)\right)^{\prime}-\beta\left(u \gamma_{k}^{\prime}(u)-\gamma_{k}(u)\right)+2 \beta\left(w \gamma_{k}^{\prime}(u)-\gamma_{k}(w)\right),
\end{aligned}
$$

and

$$
D=\left(w \gamma_{k}(w)\right)^{\prime}-\beta\left(\gamma_{k}(w)-w \gamma_{k}^{\prime}(w)\right) \text {. }
$$

Using the formulas (5.3), (5.4), (5.5) and (5.6), a computation gives the following lemma.

Lemma 5.1. For $(s, u, w) \in R(t)$,

$$
\begin{aligned}
\left|J_{\varphi_{t}}(s, u, w)\right| & \leq C \gamma_{k}^{\prime}(s) \gamma_{k}^{\prime}(w), \\
\left|\frac{\partial}{\partial s}\left(J_{\varphi_{t}}\right)\right| & \leq C \gamma_{k}^{\prime \prime}(s) \gamma_{k}^{\prime}(w), \\
\left|\frac{\partial}{\partial u}\left(J_{\varphi_{t}}\right)\right| & \leq C \gamma_{k}^{\prime}(s)\left(\gamma_{k}^{\prime \prime}(u)+\gamma_{k}^{\prime}(w)\right),
\end{aligned}
$$

and

$$
\left|\frac{\partial}{\partial w}\left(J_{\varphi_{t}}\right)\right| \leq C \gamma_{k}^{\prime \prime}(w) \gamma_{k}^{\prime}(s) .
$$

We now turn our attention to estimating $J_{\varphi_{t}}$ from below. 
Lemma 5.2. For $1 \leq u<w \leq 2$, there are three functions $s_{1}(u, w), s_{2}(u, w)$ and $s_{3}(u, w)$ such that for $(s, u, w) \in R(t)$,

$$
\left|J_{\varphi_{t}}(s, u, w)\right| \geq \epsilon \gamma_{k}^{\prime}(s) \gamma_{k}^{\prime}(w)(w-u)\left|s-s_{1}\right|\left|s-s_{2}\right|\left|s-s_{3}\right|
$$

for some $\epsilon>0$.

Proof. For a fixed $(u, w), u<w$, consider

$$
f(s)=J_{\varphi_{t}}(s, u, w) .
$$

Expanding (4.3) on the first column, we see that

$f(s)=a(u, w)+b(u, w) \gamma_{k}^{\prime}(s)+\left(\gamma_{k}^{\prime}(w)-\gamma_{k}^{\prime}(u)\right)\left((\beta-1) s \gamma_{k}^{\prime}(s)-(\beta+1) \gamma_{k}(s)\right)$.

Consider the case $\beta \neq 1$. Then

$$
\left(\frac{f^{\prime}(s)}{\gamma_{k}^{\prime \prime}(s)}\right)^{\prime}=\left(\gamma_{k}^{\prime}(w)-\gamma_{k}^{\prime}(u)\right)\left[(\beta-1)-2\left(\frac{s}{\lambda\left(2^{-k} s\right)}\right)^{\prime}\right] .
$$

Since

$$
\left(\frac{s}{\lambda\left(2^{-k} s\right)}\right)^{\prime}=\frac{1}{\lambda\left(2^{-k} s\right)}-\frac{2^{-k} s \lambda^{\prime}\left(2^{-k} s\right)}{\lambda^{2}\left(2^{-k} s\right)}
$$

is arbitrarily small by (7) and the assumption that $\lambda$ is large, we see that $f^{\prime}(s) / \gamma_{k}^{\prime \prime}(s)$ is monotone and thus $f^{\prime}(s)$ has at most one zero, $s_{1}=s_{1}(u, w)$, and $f(s)$ has at most two zeros, $s_{2}=s_{2}(u, w)$ and $s_{3}=s_{3}(u, w)$. We will assume that $f^{\prime}$ has one zero and $f$ has two zeros with $s_{2}<s_{1}<s_{3}$, otherwise the proof is easier. From (5.11), we have

$$
\left|f^{\prime}(s)\right| \geq \epsilon\left(\gamma_{k}^{\prime}(w)-\gamma_{t}^{\prime}(u)\right) \gamma_{k}^{\prime \prime}(s)\left|s-s_{1}\right|
$$

for some $\epsilon>0$. Suppose $s_{1} \leq s \leq s_{3}$. Then

$$
\begin{aligned}
|f(s)| & =\left|\int_{s}^{s_{3}} f^{\prime}(r) d s\right| \\
& \geq \epsilon\left(\gamma_{k}^{\prime}(w)-\gamma_{k}^{\prime}(u)\right) \int_{s}^{s_{3}} \gamma_{k}^{\prime \prime}(r)\left(r-s_{1}\right) d r \\
& \geq \epsilon\left(\gamma_{k}^{\prime}(w)-\gamma_{k}^{\prime}(u)\right)\left(\gamma_{k}^{\prime}\left(s_{3}\right)-\gamma_{k}^{\prime}(s)\right)\left(s-s_{1}\right)
\end{aligned}
$$

and so the conclusion follows from Lemma 4.5. Next suppose $s_{3} \leq s$. Then as before

$$
\begin{aligned}
|f(s)| & \geq \epsilon\left(\gamma_{k}^{\prime}(w)-\gamma_{k}^{\prime}(u)\right) \int_{s_{3}}^{s} \gamma_{k}^{\prime \prime}(r)\left(r-s_{1}\right) d r \\
& \geq \epsilon\left(\gamma_{k}^{\prime}(w)-\gamma_{k}^{\prime}(u)\right) \int_{\frac{s_{3}+s}{2}}^{s} \gamma_{k}^{\prime \prime}(r)\left(r-s_{1}\right) d r \\
& \geq \frac{\epsilon}{2}\left(\gamma_{k}^{\prime}(w)-\gamma_{k}^{\prime}(u)\right)\left(\gamma_{k}^{\prime}(s)-\gamma_{k}^{\prime}\left(\frac{s+s_{3}}{2}\right)\right)\left(s-s_{1}\right)
\end{aligned}
$$

and so the conclusion follows from Lemma 4.5. The case $s \leq s_{1}$ is treated similarly. When $\beta=1$, one can consider

$$
\left(\frac{f^{\prime}(s)}{\gamma_{k}^{\prime}(s)}\right)^{\prime}=b(u, w)\left(\frac{\lambda\left(2^{-k} s\right)}{s}\right)^{\prime} \text {. }
$$


Then one can estimate the coefficient $b(u, w)$ from below and proceed as above. This concludes the proof of the lemma.

Now by Fubini's theorem we have

Lemma 5.3. The zero set of $J_{\varphi_{t}}$ has measure zero.

We now obtain another estimate for $J_{\varphi_{t}}$ from below on $\Omega_{k}^{1}$.

Lemma 5.4. For $(s, u, w) \in \Omega_{k}^{1}$,

$$
\left|J_{\varphi_{t}}(s, u, w)\right| \geq \frac{1}{2} \gamma_{k}^{\prime}(s) \gamma_{k}^{\prime}(w)\left|P_{t}(s, u, w)\right| .
$$

Proof. Expanding (5.3), we see that for $(s, u, w) \in \Omega_{k}^{1}$,

$$
\begin{aligned}
& J_{\varphi_{t}}(s, u, w)=- \gamma_{k}^{\prime}(s) \gamma_{k}^{\prime}(w) P_{t}(s, u, w) \\
&+\mathscr{O}\left(\gamma_{k}^{\prime}(s)\left(\gamma_{k}^{\prime}(u)+\gamma_{k}(w)\right)+\gamma_{k}(s) \gamma_{k}^{\prime}(w)\right) \\
&=-\gamma_{k}^{\prime}(s) \gamma_{k}^{\prime}(w) P_{t}(s, u, w)+\mathscr{O}\left(\frac{\gamma_{k}^{\prime}(s) \gamma_{k}^{\prime}(w)}{\lambda\left(2^{-k} s\right)}\right) .
\end{aligned}
$$

The last equality follows from Lemmas 4.2 and 4.3. Since $\left|P_{t}(s, u, w)\right| \geq$ $C \frac{1}{\lambda\left(2^{-k} s\right)}$ for $(s, u, w) \in \Omega_{k}^{1}$, the desired estimate follows if $C$ is chosen large enough.

One consequence of the lemmas in Sections 4 and 5 are certain estimates for the functions $r_{1}, r_{2}, r_{3}, \tilde{r}_{1}, \tilde{r}_{2}$ and $\tilde{r}_{3}$.

Lemma 5.5. Let $\delta>0$. Then if $\epsilon$ in the definition of $r_{1}, r_{2}, r_{3}$, etc. is sufficiently small, and $p=(s, u, w) \in R(t)$,

$$
\begin{aligned}
& r_{1}(p)=\tilde{r}_{1}(p) \leq \delta(s-t) \frac{1}{\lambda\left(2^{-k} s\right)}, \\
& r_{2}(p)=r_{3}(p)=\tilde{r}_{2}(p) \leq \delta(w-u) \frac{1}{\lambda\left(2^{-k} w\right)},
\end{aligned}
$$

and for $p \in \Omega_{k}^{3}$,

$$
\tilde{r}_{3}(p) \leq \delta(w-u) \frac{1}{\lambda\left(2^{-k} s\right)} .
$$

Proof. The inequalities follow easily from (5.12). In particular for $p \in \Omega_{k}^{3}$, we have $\left|F_{t}(s, u, w)\right| \leq C / \lambda\left(2^{-k} s\right)$ and so (5.12) shows $\left|J_{\varphi_{t}}(p)\right| \leq C \frac{\gamma_{k}^{\prime}(s) \gamma_{k}^{\prime}(w)}{\lambda\left(2^{-k} s\right)}$ which implies (5.15).

Lemma 5.6. There are positive constants $C_{1}$ and $C_{2}$ such that if $p=(s, u, w) \in$ $\Omega_{k}^{2}$ and $q \in D^{*}(p)$,

$$
C_{1} \leq \frac{\left|J_{\varphi_{t}}(q)\right|}{\left|J_{\varphi_{t}}(p)\right|} \leq C_{2} .
$$


Proof.

$$
\begin{aligned}
\left|J_{\varphi_{t}}(q)-J_{\varphi_{t}}(p)\right|= & \left|\int_{0}^{1} \nabla J_{\varphi_{t}}(\tau q+(1-\tau) p) \cdot(q-p) d \tau\right| \\
\leq & C r_{1}(p) \int_{0}^{1}\left|\frac{\partial}{\partial s} J_{\varphi_{t}}(\tau q+(1-\tau) p)\right| d \tau \\
& +C r_{2}(p) \int_{0}^{1}\left|\frac{\partial}{\partial w} J_{\varphi_{t}}(\tau q+(1-\tau) p)\right| d \tau \\
& +C r_{3}(p) \int_{0}^{1}\left|\frac{\partial}{\partial u} J_{\varphi_{t}}(\tau q+(1-\tau) p)\right| d \tau .
\end{aligned}
$$

Using Lemmas 5.1, 5.5 and 4.4, we see that

$$
\begin{aligned}
r_{1}(p) \int_{0}^{1}\left|\frac{\partial}{\partial s} J_{\varphi_{t}}(\tau q+(1-\tau) p)\right| d \tau & \leq C r_{1}(p) \gamma_{k}^{\prime \prime}(s) \gamma_{k}^{\prime}(w) \\
& \leq \epsilon\left|J_{\varphi_{t}}(p)\right|
\end{aligned}
$$

for $\epsilon>0$ small. Similar remarks apply to the other partial derivatives of $J_{\varphi_{t}}$ and we conclude that

$$
\left|J_{\varphi_{t}}(q)-J_{\varphi_{t}}(p)\right| \leq \frac{1}{2}\left|J_{\varphi_{t}}(p)\right|
$$

which implies the desired result by taking $C_{1}=\frac{1}{2}$ and $C_{2}=\frac{3}{2}$.

Lemma 5.7. There are positive constants $C_{1}$ and $C_{2}$ such that if $p \in \Omega_{k}^{3}$ and $q \in G^{*}(p)$,

$$
C_{1} \leq \frac{\left|J_{\varphi_{t}}(q)\right|}{\left|J_{\varphi_{t}}(p)\right|} \leq C_{2} .
$$

Proof. The proof is similar to the proof of Lemma 5.6. We should note that for $p=(s, u, w) \in \Omega_{k}^{3}$,

$$
w-u \geq C \frac{\log \lambda\left(2^{-k} u\right)}{\lambda\left(2^{-k} u\right)}
$$

and so by Lemma 4.2 ,

$$
\gamma_{k}^{\prime \prime}(u) \leq C \gamma_{k}^{\prime}(w)
$$

Therefore from Lemmas 5.1, 5.5 and 4.4,

$$
\begin{aligned}
\tilde{r}_{3}(p)\left|\frac{\partial}{\partial u} J_{\varphi_{t}}(q)\right| & \leq C \tilde{r}_{3}(p) \gamma_{k}^{\prime}(s) \gamma_{k}^{\prime}(w) \\
& \leq \epsilon\left|J_{\varphi_{t}}(p)\right|
\end{aligned}
$$

for $\epsilon>0$ small and for any $q \in G^{*}(p)$. The rest of the argument is the same as in Lemma 5.6. 
Consequently, from the proofs of Lemmas 5.6 and 5.7 we have the following estimates. For $p \in \Omega_{k}^{2}$ and $q \in D^{*}(p)$,

$$
\begin{aligned}
& \left|\frac{\partial}{\partial s} J_{\varphi_{t}}(q)\right| r_{1}(p) \leq C\left|J_{\varphi_{t}}(q)\right|, \\
& \left|\frac{\partial}{\partial w} J_{\varphi_{t}}(q)\right| r_{2}(p) \leq C\left|J_{\varphi_{t}}(q)\right|, \\
& \left|\frac{\partial}{\partial u} J_{\varphi_{t}}(q)\right| r_{3}(p) \leq C\left|J_{\varphi_{t}}(q)\right| .
\end{aligned}
$$

Also for $p \in \Omega_{k}^{3}$ and $q \in G^{*}(p)$,

$$
\begin{aligned}
& \left|\frac{\partial}{\partial s} J_{\varphi_{t}}(q)\right| \tilde{r}_{1}(p) \leq C\left|J_{\varphi_{t}}(q)\right|, \\
& \left|\frac{\partial}{\partial w} J_{\varphi_{t}}(q)\right| \tilde{r}_{2}(p) \leq C\left|J_{\varphi_{t}}(q)\right|, \\
& \left|\frac{\partial}{\partial u} J_{\varphi_{t}}(q)\right| \tilde{r}_{3}(p) \leq C\left|J_{\varphi_{t}}(q)\right| .
\end{aligned}
$$

Lemma 5.8. There are positive constants $C_{1}$ and $C_{2}$ such that if $p \in \Omega_{k}^{2}$ and $q \in D^{*}(p)$,

$$
C_{1} \leq \frac{r_{j}(q)}{r_{j}(p)} \leq C_{2}, \quad j=1,2,3 .
$$

Also if $p \in \Omega_{k}^{3}$ and $q \in G^{*}(p)$, we have

$$
C_{1} \leq \frac{\tilde{r}_{j}(q)}{\tilde{r}_{j}(p)} \leq C_{2}, \quad j=1,2,3 .
$$

Proof. Let $p=(s, u, w)$ and $q=\left(s_{1}, u_{1}, w_{1}\right)$. Since $r_{1}(p) \leq \delta(s-t)$ by (5.13), it is clear that $C_{1}(s-t) \leq\left(s_{1}-t\right) \leq C_{2}(s-t)$ for some positive constants $C_{1}$ and $C_{2}$. Similar remarks apply to $w-u, \operatorname{dist}(p, X)$ and $\operatorname{dist}(p, Y)$ and therefore we can find constants $C_{1}$ and $C_{2}$ such that

$$
C_{1} \leq \frac{\Delta(q)}{\Delta(p)} \leq C_{2}
$$

for $q \in D^{*}(p)$ if $p \in \Omega_{k}^{2}$ and $q \in G^{*}(p)$ if $p \in \Omega_{k}^{3}$. Furthermore by Lemmas 5.5 and 4.4 we have similar estimates for the functions $\gamma_{k}^{\prime}$ and $\gamma_{k}^{\prime \prime}$. Now applying Lemmas 5.6 and 5.7 completes the proof of Lemma 5.8.

To end this section we will collect some of the estimates proved so far and put them in a form which will be useful in proving the estimate (1.2). Recall that to prove (1.2) we need to have estimates on $\frac{g_{i j}}{\operatorname{det} \mathscr{G}}=\frac{g_{i j}}{J_{i t}}$ where $g_{i j}$ is the $(i, j)$ cofactor of the matrix $\mathscr{G}$ introduced at the end of Section 3. 
Lemma 5.9. For $p=(s, u, w) \in Q_{\ell n} \subset \Omega_{k}^{1}$,

$$
\begin{aligned}
& \left|\frac{g_{1 j}(p)}{\operatorname{det} \mathscr{G}(p)}\right| \leq C 2^{\ell}, \quad j=1,2,3, \\
& \left|g_{11}(p) \frac{\partial}{\partial s}\left(\frac{1}{\operatorname{det} \mathscr{G}}\right)(p)\right| \leq C 2^{2 \ell} \frac{\gamma_{k}^{\prime \prime}(s)}{\gamma_{k}^{\prime}(s)^{2}} \gamma_{k}^{\prime}(w), \\
& \left|g_{12}(p) \frac{\partial}{\partial u}\left(\frac{1}{\operatorname{det} \mathscr{G}}\right)(p)\right| \leq C 2^{\ell}\left|\frac{g_{12}}{\operatorname{det} \mathscr{G}}(p)\right|,
\end{aligned}
$$

and

$$
\left|g_{13}(p) \frac{\partial}{\partial w}\left(\frac{1}{\operatorname{det} \mathscr{G}}\right)(p)\right| \leq C 2^{2 \ell} .
$$

For $p \in D^{*}\left(p_{0}\right) \subset \Omega_{k}^{2}$,

$$
\begin{aligned}
& \left|\frac{g_{11}}{\operatorname{det} \mathscr{G}}(p)\right| \leq C \frac{1}{r_{3}(p) \lambda\left(2^{-k} w\right)}, \\
& \left|g_{11}(p) \frac{\partial}{\partial s}\left(\frac{1}{\operatorname{det} \mathscr{G}}\right)(p)\right| \leq C \frac{1}{r_{1}(p)}\left|\frac{g_{11}}{\operatorname{det} \mathscr{G}}(p)\right|, \\
& \left|\frac{g_{12}}{\operatorname{det} \mathscr{G}}(p)\right| \leq C \frac{1}{r_{1}(p) \lambda\left(2^{-k} s\right)}, \\
& \left|g_{12}(p) \frac{\partial}{\partial u}\left(\frac{1}{\operatorname{det} \mathscr{G}}\right)(p)\right| \leq C \frac{1}{r_{3}(p)}\left|\frac{g_{12}}{\operatorname{det} \mathscr{G}}(p)\right|, \\
& \left|\frac{g_{13}}{\operatorname{det} \mathscr{G}}(p)\right| \leq C \frac{1}{r_{1}(p)} \frac{1}{\lambda\left(2^{-k} s\right)},
\end{aligned}
$$

and

$$
\left|g_{13}(p) \frac{\partial}{\partial w}\left(\frac{1}{\operatorname{det} \mathscr{G}}\right)(p)\right| \leq C \frac{1}{r_{2}(p)}\left|\frac{g_{13}}{\operatorname{det} \mathscr{G}}(p)\right| .
$$

And for $p \in G^{*}\left(p_{0}\right) \subset \Omega_{k}^{3}$,

$$
\begin{aligned}
& \left|\frac{g_{11}}{\operatorname{det} \mathscr{G}}(p)\right| \leq C \frac{1}{\tilde{r}_{3}(p)}, \\
& \left|g_{11}(p) \frac{\partial}{\partial s}\left(\frac{1}{\operatorname{det} \mathscr{G}}\right)(p)\right| \leq C \frac{1}{\tilde{r}_{1}(p)}\left|\frac{g_{11}}{\operatorname{det} \mathscr{G}}(p)\right|, \\
& \left|\frac{g_{12}}{\operatorname{det} \mathscr{G}}(p)\right| \leq C \frac{1}{\tilde{r}_{1}(p)}, \\
& \left|g_{12}(p) \frac{\partial}{\partial u}\left(\frac{1}{\operatorname{det} \mathscr{G}}\right)(p)\right| \leq C \frac{1}{\tilde{r}_{3}(p)}\left|\frac{g_{12}}{\operatorname{det} \mathscr{G}}(p)\right|,
\end{aligned}
$$

and

$$
\left|g_{13}(p) \frac{\partial}{\partial w}\left(\frac{1}{\operatorname{det} \mathscr{G}}\right)(p)\right| \leq C \frac{1}{\tilde{r}_{2}(p)}\left|\frac{g_{13}}{\operatorname{det} \mathscr{G}}(p)\right|
$$


Proof. Using (5.3) we may write

and

$$
\begin{aligned}
& g_{11}(p)=C \gamma_{k}^{\prime}(w)-D \gamma_{k}^{\prime}(u), \\
& g_{12}(p)=D \gamma_{k}^{\prime}(s)-A \gamma_{k}^{\prime}(w),
\end{aligned}
$$

$$
g_{13}(p)=A \gamma_{k}^{\prime}(u)-C \gamma_{k}^{\prime}(s)
$$

where $A, C$ and $D$ are written out in (5.5), (5.6) and (5.7). For $p=$ $(s, u, w) \in R(t)$, we easily see that

$$
\left|g_{1 j}(p)\right| \leq C \gamma_{k}^{\prime}(s) \gamma_{k}^{\prime}(w), \quad j=1,2,3 .
$$

However by examining $g_{13}(p)$, we actually have the estimate

$$
\left|g_{13}(p)\right| \leq C \gamma_{k}^{\prime}(s)\left(\gamma_{k}^{\prime}(u)+\gamma_{k}(w)\right) \text {. }
$$

Therefore if $p \in \Omega_{k}^{1} \cup \Omega_{k}^{3}$,

$$
\left|g_{13}(p)\right| \leq C \frac{\gamma_{k}^{\prime}(s) \gamma_{k}^{\prime}(w)}{\lambda\left(2^{-k} w\right)}
$$

In fact for $p=(s, u, w) \in \Omega_{k}^{1} \cup \Omega_{k}^{3}$,

$$
w-u \geq C \frac{\log \lambda\left(2^{-k} u\right)}{\lambda\left(2^{-k} u\right)}
$$

and so Lemmas 4.2 and 4.3 imply (5.39).

Now using the estimate from below on $\operatorname{det} \mathscr{G}(p)=J_{\varphi_{t}}(p)$ for $p \in \Omega_{k}^{1}$ obtained in Lemma 5.4, together with (5.38), gives us (5.22). In fact for $p=(s, u, w) \in Q_{\ell n},\left|P_{t}(s, u, w)\right| \geq \epsilon 2^{-\ell}$ for some $\epsilon>0$. With these observations, (5.23), (5.24) and (5.25) follow from a straightforward calculation, using (5.38), (5.39) and Lemma 5.1. Also from (5.38), the estimates contained in (5.26), (5.28), (5.30), (5.32) and (5.34) follow directly from the definitions of $r_{1}, r_{2}, r_{3}$, etc. Estimate (5.36) follows in a similar fashion from (5.39). Finally (5.27), (5.29), (5.31), (5.33) and (5.35) are consequences of the estimates (5.16)-(5.21). This completes the proof.

\section{THE ONE TO ONE PROPERTY OF $\varphi_{t}$}

In this section we prove that $\varphi_{t}$ is one to one on each ball $E(p)$.

Lemma 6.1. Let $A, B, C$ and $X$ be vectors in $\mathbb{R}^{3}$. Then

$$
\operatorname{det}(A, B,[X, C])=\operatorname{det}([A, B], X, C)
$$

and

$$
\operatorname{det}([A, X], B, C)+\operatorname{det}(A,[B, X], C)+\operatorname{det}(A, B,[C, X])=0 .
$$

Proof. The proof can be established by direct computation.

Lemma 6.2. $\varphi_{t}$ is one to one on each $B(p)$. 
Proof. We shall show that $\varphi_{t}\left(s_{1}, u_{1}, w_{1}\right) \neq \varphi_{t}\left(s_{2}, u_{2}, w_{2}\right)$ for any two distinct points $\left(s_{1}, u_{1}, w_{1}\right)$ and $\left(s_{2}, u_{2}, w_{2}\right)$ in $B(p)$. We will further assume $s_{1} \neq s_{2}$, $u_{1} \neq u_{2}$ and $w_{1} \neq w_{2}$. If two of the coordinates of the distinct points are the same, the proof follows trivially from the convexity of $\gamma(t)$. Now

$$
\begin{aligned}
\varphi_{t}\left(s_{1},\right. & \left.u_{1}, w_{1}\right)-\varphi_{t}\left(s_{2}, u_{2}, w_{2}\right) \\
= & \varphi_{t}\left(s_{1}, u_{1}, w_{1}\right)-\varphi_{t}\left(s_{2}, u_{1}, w_{1}\right) \\
& +\varphi_{t}\left(s_{2}, u_{1}, w_{1}\right)-\varphi_{t}\left(s_{2}, u_{2}, w_{1}\right) \\
& +\varphi_{t}\left(s_{2}, u_{2}, w_{1}\right)-\varphi_{t}\left(s_{2}, u_{2}, w_{2}\right) \\
= & I+I I+I I I
\end{aligned}
$$

where

$$
\begin{aligned}
I= & \Gamma_{k}\left(s_{1}\right)-\Gamma_{k}\left(s_{2}\right) \\
& +\beta\left[\Gamma_{k}\left(s_{1}\right)-\Gamma_{k}\left(s_{2}\right),-\Gamma_{k}(t)+\Gamma_{k}\left(u_{1}\right)-\Gamma_{k}\left(w_{1}\right)\right], \\
I I= & \Gamma_{k}\left(u_{1}\right)-\Gamma_{k}\left(u_{2}\right) \\
& +\beta\left[\Gamma_{k}\left(u_{1}\right)-\Gamma_{k}\left(u_{2}\right),-\Gamma_{k}\left(s_{2}\right)+\Gamma_{k}(t)-\Gamma_{k}\left(w_{1}\right)\right],
\end{aligned}
$$

and

$$
\begin{aligned}
I I I= & \Gamma_{k}\left(w_{2}\right)-\Gamma_{k}\left(w_{1}\right) \\
& +\beta\left[\Gamma_{k}\left(w_{2}\right)-\Gamma_{k}\left(w_{1}\right),-\Gamma_{k}\left(s_{2}\right)+\Gamma_{k}(t)-\Gamma_{k}\left(u_{2}\right)\right] .
\end{aligned}
$$

It suffices to prove $I, I I$ and $I I I$ are linearly independent, and hence it suffices to show that $\operatorname{det}(I, I I, I I I) \neq 0$. Let $\alpha\left(s_{1}, s_{2}, u_{1}, u_{2}, w_{1}, w_{2}\right)=\operatorname{det}(I, I I, I I I)$. Then $\alpha\left(s, s, u_{1}, u_{2}, w_{1}, w_{2}\right)=0$ and so we may write either

$$
\begin{aligned}
& \alpha\left(s_{1}, s_{2}, u_{1}, u_{2}, w_{1}, w_{2}\right) \\
& =-\int_{s_{1}}^{s_{2}} \frac{\partial \alpha}{\partial s_{1}}\left(s, s_{2}, u_{1}, u_{2}, w_{1}, w_{2}\right) d s
\end{aligned}
$$

or

$$
\begin{aligned}
& \alpha\left(s_{1}, s_{2}, u_{1}, u_{2}, w_{1}, w_{2}\right) \\
& \quad=\int_{s_{1}}^{s_{2}} \frac{\partial \alpha}{\partial s_{2}}\left(s_{1}, s, u_{1}, u_{2}, w_{1}, w_{2}\right) d s .
\end{aligned}
$$

Furthermore, $\alpha\left(s_{1}, s_{2}, u, u, w_{1}, w_{2}\right)=0$ so

$$
\frac{\partial \alpha}{\partial s_{1}}\left(s, s_{2}, u, u, w_{1}, w_{2}\right)=0
$$

and

$$
\frac{\partial \alpha}{\partial s_{2}}\left(s_{1}, s, u, u, w_{1}, w_{2}\right)=0 .
$$

Thus we may use the reasoning establishing (6.5) and (6.6) to write $\alpha$ as a double integral involving

$$
\frac{\partial^{2} \alpha}{\partial s_{1} \partial u_{1}}, \frac{\partial^{2} \alpha}{\partial s_{1} \partial u_{2}}, \frac{\partial^{2} \alpha}{\partial s_{2} \partial u_{1}} \text { or } \frac{\partial^{2} \alpha}{\partial s_{2} \partial u_{2}} \text {. }
$$


After one more iteration we find

$$
\begin{aligned}
& \alpha\left(s_{1}, s_{2}, u_{1}, u_{2}, w_{1}, w_{2}\right) \\
& \quad= \pm \int_{s_{1}}^{s_{2}} \int_{u_{1}}^{u_{2}} \int_{w_{1}}^{w_{2}} \frac{\partial^{3} \alpha}{\partial s_{a} \partial u_{b} \partial w_{c}} d s d u d w
\end{aligned}
$$

where each of $a, b$ and $c$ can be chosen to be either 1 or 2 . Thus it suffices to show that for some choice of $(a, b, c)$

$$
\frac{\partial^{3}}{\partial s_{a} \partial u_{b} \partial w_{c}} \operatorname{det}(I, I I, I I I) \neq 0
$$

in $B(p)$.

The computation of $\frac{\partial^{3}}{\partial s_{a} \partial u_{b} \partial w_{c}} \operatorname{det}(I, I I, I I I)$ is quite tedious, but can be somewhat simplified using Lemma 6.1. In order to express the result we use the notation that if $a=2, a^{\prime}=1$ and if $a=1, a^{\prime}=2$, and similarly with $b$ and $c$. Then we find

$$
\begin{aligned}
& \pm \frac{\partial^{3}}{\partial s_{a} \partial u_{b} \partial w_{c}} \operatorname{det}(I, I I, I I I) \\
& \quad=J_{\varphi_{t}}\left(s_{a}, u_{b}, w_{c}\right) \\
& \quad+\beta \operatorname{det}\left(\left[\Gamma_{k}\left(s_{a}\right)-\Gamma_{k}\left(s_{a^{\prime}}\right), \Gamma_{k}^{\prime}\left(s_{a}\right)\right], \Gamma_{k}^{\prime}\left(u_{b}\right),-\Gamma_{k}^{\prime}\left(w_{c}\right)\right) \\
& \quad+\beta \operatorname{det}\left(\Gamma_{k}^{\prime}\left(s_{a}\right), \Gamma_{k}^{\prime}\left(u_{b}\right),\left[\Gamma_{k}^{\prime}\left(w_{c}\right), \Gamma_{k}\left(w_{c}\right)-\Gamma_{k}\left(w_{c^{\prime}}\right)\right]\right)
\end{aligned}
$$

More explicitly,

$$
\begin{aligned}
& \pm \frac{\partial^{3}}{\partial s_{a} \partial u_{b} \partial w_{c}} \operatorname{det}(I, I I I, I I I) \\
& \quad=J_{\varphi_{t}}\left(s_{a}, u_{b}, w_{c}\right) \\
& \quad+\beta\left(\gamma_{k}^{\prime}\left(s_{a}\right)\left(s_{a}-s_{a^{\prime}}\right)-\left(\gamma_{k}\left(s_{a}\right)-\gamma_{k}\left(s_{a^{\prime}}\right)\right)\right)\left(\gamma_{k}^{\prime}\left(u_{b}\right)-\gamma_{k}^{\prime}\left(w_{c}\right)\right) \\
& \quad+\beta\left(\gamma_{k}^{\prime}\left(w_{c}\right)\left(w_{c}-w_{c^{\prime}}\right)-\left(\gamma_{k}\left(w_{c}\right)-\gamma_{k}\left(w_{c^{\prime}}\right)\right)\right)\left(\gamma_{k}^{\prime}\left(s_{a}\right)-\gamma_{k}^{\prime}\left(u_{b}\right)\right) .
\end{aligned}
$$

Choose $a$ and $c$ so that $s_{a^{\prime}}<s_{a}$ and $w_{c^{\prime}}<w_{c}$, i.e., if $s_{1}<s_{2}$ choose $a=2$, and if $s_{2}<s_{1}$ choose $a=1$. Then if $p \in Q_{\ell n}^{*} \subset \Omega_{k}^{1}$,

$$
\left|\frac{\partial^{3} \operatorname{det}(I, I I, I I I)}{\partial s_{a} \partial u_{b} \partial w_{c}}\right|=\left|J_{\varphi_{t}}\left(s_{a}, u_{b}, w_{c}\right)\right|+E,
$$

where

$$
|E| \leq C \operatorname{diam}\left(Q_{\ell n}\right) \gamma_{k}^{\prime}\left(s_{a}\right) \gamma_{k}^{\prime}\left(w_{c}\right) .
$$

Recall that for any point $p=(s, u, w) \in Q_{\ell n}^{*}$,

$$
\left|P_{t}(s, u, w)\right| \geq \epsilon 2^{-\ell} \text { for some } \epsilon>0 .
$$

Therefore according to Lemma 5.4,

$$
\left|J_{\varphi_{t}}\left(s_{a}, u_{b}, w_{c}\right)\right| \geq \epsilon 2^{-\ell} \gamma_{k}^{\prime}\left(s_{a}\right) \gamma_{k}^{\prime}\left(w_{c}\right) .
$$


Now if we choose $\epsilon_{0}$ in the definition of $Q_{\ell n}$ sufficiently small, (6.10) and (6.11) imply $\operatorname{det}(I, I I, I I I) \neq 0$ on $Q_{\ell n}^{*}$.

Next suppose $p=(s, u, w) \in \Omega_{k}^{2}$. Then we obtain from (6.9),

$$
\left|\frac{\partial^{3} \operatorname{det}(I, I I, I I I)}{\partial s_{a} \partial u_{b} \partial w_{c}}\right|=\left|J_{\varphi_{t}}\left(s_{a}, u_{b}, w_{c}\right)\right|+E
$$

where

$$
|E| \leq C \gamma_{k}^{\prime}\left(s_{a}\right) \gamma_{k}^{\prime}\left(w_{c}\right)\left(r_{1}(p)+r_{2}(p)\right)
$$

By Lemma 5.8,

$$
|E| \leq c \gamma_{k}^{\prime}\left(s_{a}\right) \gamma_{k}^{\prime}\left(w_{c}\right)\left(r_{1}\left(s_{a}, u_{b}, w_{c}\right)+r_{2}\left(s_{a}, u_{b}, w_{c}\right)\right) .
$$

Hence from the definitions of $r_{1}$ and $r_{2},(3.10)$ and (3.11), we see that

$$
\frac{\partial^{3} \operatorname{det}(I, I I, I I I)}{\partial s_{a} \partial u_{b} \partial w_{c}} \neq 0
$$

on $B(p)$ if $\epsilon$ in (3.10) and (3.11) is chosen sufficiently small. The same reasoning gives (6.14) on $B(p)$ where $p \in \Omega_{k}^{3}$. This completes the proof that $\varphi_{t}$ is one to one on each ball $B(p)$.

\section{A PARTITION OF UNITY}

We wish to find a sequence of points $p_{1}, p_{2}, p_{3}, \ldots$ in $R(t)$, balls $B\left(p_{1}\right)$, $B\left(p_{2}\right), \ldots$, and corresponding functions $\psi_{1}, \psi_{2}, \ldots$ which form a suitable partition of unity.

Lemma 7.1. There exists a sequence of points $\left\{p_{j}\right\}$ in $R(t)$ such that except for a set of measure zero (the zero set of $J_{\varphi_{t}}$ ),

$$
R(t)=\cup B\left(p_{j}\right) .
$$

There is a constant $C$ so that no point $x$ is in more than $C$ of the balls $B\left(p_{j}\right)$.

Moreover there exists nonnegative $C^{\infty}$ functions $\psi_{j}$ such that

(7.3) support of $\psi_{j} \subset B\left(p_{j}\right)$.

(7.4) $\sum \psi_{j} \equiv 1$, except on a set of measure zero.

(7.5) If $p_{j} \in Q_{\ell n}^{*} \subset \Omega_{k}^{1}$,

$$
\left\|\nabla \psi_{j}\right\|_{L^{\infty}} \leq C 2^{-\ell}
$$

(7.6) If $p_{j} \in \Omega_{k}^{2}$,

$$
\left\|\frac{\partial \psi_{j}}{\partial s}\right\|_{L^{\infty}} \leq C \frac{1}{r_{1}\left(p_{j}\right)}, \quad\left\|\frac{\partial \psi_{j}}{\partial w}\right\|_{L^{\infty}} \leq C \frac{1}{r_{2}\left(p_{j}\right)},
$$

and

$$
\left\|\frac{\partial \psi_{j}}{\partial u}\right\|_{L^{\infty}} \leq C \frac{1}{r_{3}\left(p_{j}\right)}
$$


(7.7) If $p_{j} \in \Omega_{k}^{3}$,

$$
\left\|\frac{\partial \psi_{j}}{\partial s}\right\|_{L^{\infty}} \leq C \frac{1}{\tilde{r}_{1}\left(p_{j}\right)}, \quad\left\|\frac{\partial \psi_{j}}{\partial w}\right\|_{L^{\infty}} \leq C \frac{1}{\tilde{r}_{2}\left(p_{j}\right)},
$$

and

$$
\left\|\frac{\partial \psi_{j}}{\partial u}\right\|_{L^{\infty}} \leq C \frac{1}{\tilde{r}_{3}\left(p_{j}\right)} .
$$

Lemma 7.1 follows from the next lemma.

Lemma 7.2. There exists a sequence of points $p_{j}$ in $\Omega_{k}^{2}$ such that $\cup D\left(p_{j}\right)=\Omega_{k}^{2}$ and every point is in at most $C$ of the $D^{*}\left(p_{j}\right)$. Also there is a sequence of points $p_{j}$ in $\Omega_{k}^{3}$ such that $\cup G\left(p_{j}\right)=\Omega_{k}^{3}$ and no point is in more than $C$ of the $G^{*}\left(p_{j}\right)$.

Let us note that an analogous statement for the $Q_{\ell, n}$ is well known. The proof of Lemma 7.2 follows the lines of a similar argument due to Sogge and Stein [SS].

For $\eta>0$ and $p_{0}=\left(s_{0}, u_{0}, w_{0}\right) \in \Omega_{k}^{2}$, set

$$
\begin{array}{r}
D_{*}\left(p_{0}\right)=\left\{(s, u, w) \mid s_{0}-\eta r_{1}\left(p_{0}\right)<s<s_{0}+\eta r_{1}\left(p_{0}\right),\right. \\
w_{0}-\eta r_{2}\left(p_{0}\right)<w<w_{0}+\eta r_{2}\left(p_{0}\right), \\
\left.u_{0}-\eta r_{3}\left(p_{0}\right)<u<u_{0}+\eta r_{3}\left(p_{0}\right)\right\},
\end{array}
$$

and for $p_{0} \in \Omega_{k}^{3}$, let

$$
\begin{array}{r}
G_{*}\left(p_{0}\right)=\left\{(s, u, w) \mid s_{0}-\eta \tilde{r}_{1}\left(p_{0}\right)<s<s_{0}+\eta \tilde{r}_{1}\left(p_{0}\right),\right. \\
w_{0}-\eta \tilde{r}_{2}\left(p_{0}\right)<w<w_{0}+\eta \tilde{r}_{2}\left(p_{0}\right), \\
\left.u_{0}-\eta \tilde{r}_{3}\left(p_{0}\right)<u<u_{0}+\eta \tilde{r}_{3}\left(p_{0}\right)\right\} .
\end{array}
$$

Lemma 7.2 follows from the following covering lemma.

Lemma 7.3. . If $\eta>0$ is sufficiently small, there exists a sequence $\left\{p_{j}\right\}$ in $\Omega_{k}^{2}$ such that the $D_{*}\left(p_{j}\right)$ are disjoint, $\cup D\left(p_{j}\right)$ covers $\Omega_{k}^{2}$ and no point is in more than $C$ of the $D^{*}\left(p_{j}\right)$. Similarly there exists a sequence $\left\{p_{j}\right\}$ in $\Omega_{k}^{3}$ such that the $G_{*}\left(p_{j}\right)$ are disjoint, $\cup G\left(p_{j}\right)$ covers $\Omega_{k}^{3}$ and no point is in more than $C$ of the $G^{*}\left(p_{j}\right)$.

Proof. We shall just consider the first statement of the lemma. The second statement is proved in a similar fashion. We will use Lemma 5.8 which asserts that if $q_{1}$ and $q_{2}$ are in $D^{*}(p)$ for some $p \in \Omega_{k}^{2}$, then $r_{j}\left(q_{1}\right)$ and $r_{j}\left(q_{2}\right)$ are comparble for $j=1,2$, and 3 . Taking this into account, we may use a Vitali type procedure to select balls, $D_{*}\left(p_{j}\right)$, according to the size of the measure of the $D_{*}\left(p_{j}\right)$, and find a sequence of points $\left\{p_{j}\right\}$ such that the $D_{*}\left(p_{j}\right)$ are disjoint and $\cup D\left(p_{j}\right)$ covers $\Omega_{k}^{2}$. We claim that any point $p$ is in at most $C$ of the $D^{*}\left(p_{j}\right)$. Suppose $p$ is in $N$ of the $D^{*}\left(p_{j}\right)$. Then by Lemma 5.8, we have $N$ disjoint rectangular parallelepipeds with volume $\approx \eta r_{1}(p) r_{2}(p) r_{3}(p)$ 
contained in a fixed rectangle of volume at most $C r_{1}(p) r_{2}(p) r_{3}(p)$. Clearly this puts a bound on $N$.

\section{THE PROOF OF (1.2)-THE CONTRIBUTION FROM $\Omega_{k}^{1}$}

As was pointed out in Section 1, we must show

$$
\int_{R(t)} f\left(\varphi_{t}(s, u, w)\right) d s d u d w=\int_{\mathbf{R}^{2}} f(x) \rho_{t}(x) d x
$$

where for some $\epsilon>0$ and constant $C$,

$$
\int_{\mathbb{R}^{2}}\left|\rho_{t}(x \cdot y)-\rho_{t}(x)\right| d x \leq C h^{\epsilon}
$$

if $\|y\|<h$. We shall show for $\sigma=1,2,3$,

$$
\int_{\Omega_{k}^{\sigma}} f\left(\varphi_{t}(s, u, w)\right) d s d u d w=\int_{\mathbb{R}^{2}} f(x) \rho_{t}^{\sigma}(x) d x
$$

where each $\rho_{t}^{\sigma}$ satisfies (8.1). In this section we consider the case $\sigma=1$.

Recall from Section 7 that we have functions $\psi_{\ell, n} \operatorname{such}$ that $\operatorname{supp}\left(\psi_{\ell, n}\right) \subset$ $Q_{\ell, n}^{*}, \sum \psi_{\ell, n}=1$ on $\Omega_{k}^{1}$, and

$$
\left\|\nabla \psi_{\ell, n}\right\|_{L^{\infty}} \leq C 2^{+\ell}
$$

Then

$$
\begin{array}{rl}
\int_{\Omega_{k}^{1}} & f\left(\varphi_{t}(s, u, w)\right) d s d u d w \\
& =\sum_{\ell, n} \int \psi_{\ell, n}(s, u, w) f\left(\varphi_{t}(s, u, w)\right) d s d u d w \\
& =\sum_{\ell, n} \int \rho_{\ell, n}(x) f(x) d x
\end{array}
$$

where

$$
\rho_{\ell, n}(x)=\frac{\psi_{\ell, n}\left(\varphi_{t}^{-1}(x)\right)}{\left|J_{\varphi_{t}}\left(\varphi_{t}^{-1}(x)\right)\right|} .
$$

Here we have used Lemma 6.2 to justify the above change of variables. Thus

$$
\rho_{t}^{1}=\sum_{\ell, n} \rho_{\ell, n} \text {. }
$$

To show (8.1), it suffices to show

$$
\int\left|\rho_{\ell, n}(x \cdot y)-\rho_{\ell, n}(x)\right| d x \leq C 2^{-3 \ell}
$$

and

$$
\int\left|\rho_{\ell, n}(x \cdot y)-\rho_{\ell, n}(x)\right| d x \leq C\|y\| .
$$


In fact if (8.3) and (8.4) are both valid, then

$$
\begin{aligned}
& \int\left|\rho_{t}^{1}(x \cdot y)-\rho_{t}^{1}(x)\right| d x \leq \sum_{\ell, n} \int\left|\rho_{\ell, n}(x \cdot y)-\rho_{\ell, n}(x)\right| d x \\
& \leq C \sum_{\ell \geq 0} \sum_{m \leq C 2^{2 \ell}} \min \left(2^{-3 \ell},\|y\|\right) \\
& \quad \leq C\left[\sum_{2^{-3 \ell} \leq\|y\|} 2^{-\ell}+\|y\| \sum_{2^{-3 \ell} \geq\|y\|} 2^{2 \ell}\right] \\
& \quad=C\|y\|^{1 / 3}
\end{aligned}
$$

which gives (8.1) for $\sigma=1$.

The proof of (8.3) is trivial as the left-hand side is at most

$$
\begin{aligned}
2 \int\left|\rho_{\ell, n}(x)\right| d x & =2 \int \psi_{\ell, n}(s, u, w) d s d u d w \\
& \leq 2\left|Q_{\ell n}^{*}\right| \leq C 2^{-3 \ell} .
\end{aligned}
$$

We turn to the proof of (8.4). By writing

$$
\rho_{\ell, n}(x \cdot y)-\rho_{\ell, n}(x)=\int_{0}^{1} \frac{d}{d t} \rho_{\ell, n}(x \cdot t y) d t,
$$

and using the chain rule, we find that

$$
\begin{aligned}
& \int\left|\rho_{\ell, n}(x \cdot y)-\rho_{\ell, n}(x)\right| d x \\
& \leq\left|y_{1}\right| \int_{0}^{1} \int\left|\frac{\partial \rho_{\ell, n}}{\partial x_{1}}(x \cdot t y)-\beta x_{2} \frac{\partial \rho_{\ell, n}}{\partial x_{3}}(x \cdot t y)\right| d x d t \\
& \quad+\left|y_{2}\right| \int_{0}^{1} \int\left|\frac{\partial \rho_{\ell, n}}{\partial x_{2}}(x \cdot t y)+\beta x_{1} \frac{\partial \rho_{\ell, n}}{\partial x_{3}}(x \cdot t y)\right| d x d t \\
& \quad+\left|y_{3}\right| \int_{0}^{1} \int\left|\frac{\partial \rho_{\ell, n}}{\partial x_{3}}(x \cdot t y)\right| d x d t .
\end{aligned}
$$

Replacing $x \cdot t y$ by $x$ in the inner integrals, we find that

$$
\int\left|\rho_{\ell, n}(x \cdot y)-\rho_{\ell, n}(x)\right| d x \leq C\|y\| \sum_{j=1}^{3} \int\left|L_{j}\left(\rho_{\ell, n}\right)(x)\right| d x
$$

where

and

$$
\begin{aligned}
& L_{1}=\frac{\partial}{\partial x_{1}}-\beta x_{2} \frac{\partial}{\partial x_{3}} \\
& L_{2}=\frac{\partial}{\partial x_{2}}+\beta x_{1} \frac{\partial}{\partial x_{3}}
\end{aligned}
$$

$$
L_{3}=\frac{\partial}{\partial x_{3}}
$$


are the left invariant vector fields discussed in Section 3. Of the three terms, the one corresponding to $L_{1}$ is the most difficult. To some extent this can be seen because the coordinate $x_{2}$ is unbounded in the support of $\rho_{\ell, n}$. Therefore we will restrict our attention to $L_{1}$ and in fact we will show

$$
\int\left|L_{1}\left(\rho_{\ell, n}\right)(x)\right| d x \leq C .
$$

To prove (8.5) we will first make the change of variables $x=\varphi_{t}(s, u, w)$. To do this, let us define

$$
K(s, u, w)=\frac{\psi_{\ell, n}(s, u, w)}{\left|J_{\varphi_{t}}(s, u, w)\right|}
$$

Note that $\rho_{\ell, n}(x)=K\left(\varphi_{t}^{-1}(x)\right)$. Formula (3.24) shows us how $L_{1}$ transforms under the change of variables $x=\varphi_{t}(s, u, w)$ and so we find

$$
\begin{aligned}
& \int\left|L_{1}\left(\rho_{\ell, n}\right)(x)\right| d x \\
& \quad=\int\left|\operatorname{det} \mathscr{G}(s, u, w) \|\left\langle\left(\mathscr{G}^{-1}\right)^{*} \nabla K(s, u, w), e_{1}\right\rangle\right| d s d u d w .
\end{aligned}
$$

Recall that $J_{\varphi_{t}}(s, u, w)=\operatorname{det} \mathscr{G}(s, u, w)$. By computing the first component of $\left(\mathscr{G}^{-1}\right)^{*} \nabla K(s, u, w)$, we see that

$$
\begin{aligned}
\int\left|L_{1}\left(\rho_{\ell, n}\right)(x)\right| d x \leq & \int\left|g_{11}(s, u, w) \frac{\partial K}{\partial s}\right| d s d u d w \\
& +\int\left|g_{12}(s, u, w) \frac{\partial K}{\partial u}\right| d s d u d w \\
& +\int\left|g_{13}(s, u, w) \frac{\partial K}{\partial w}\right| d s d u d w \\
= & I+I I+I I I .
\end{aligned}
$$

Using (8.2), (5.22) and (5.23), we have

$$
\begin{aligned}
I \leq C & {\left[2^{\ell} \int_{Q_{\ell, n}^{*}}\left|\frac{g_{11}}{\operatorname{det} \mathscr{G}}(p)\right| d p\right.} \\
& \left.\quad+\int_{Q_{\ell, n}^{*}}\left|g_{11}(p) \frac{\partial}{\partial s}\left(\frac{1}{\operatorname{det} \mathscr{G}}\right)(p)\right| d p\right] \\
\leq C & {\left[2^{2 \ell}\left|Q_{\ell, n}^{*}\right|+2^{2 \ell} \int_{Q_{i, n}^{*}} \frac{\gamma_{k}^{\prime \prime}(s)}{\gamma_{k}^{\prime}(s)^{2}} \gamma_{k}^{\prime}(w)\right] } \\
\leq C & {\left[2^{-\ell}+1\right] \leq C . }
\end{aligned}
$$

The estimate for the second integral follows by integrating in $s$ first and noting that every $w$ value is smaller than any $s$ value in $Q_{\ell, n}^{*}$. 
To estimate $I I$, we may use (8.2), (5.22) and (5.24) to see that

$$
\begin{aligned}
& I I \leq C {\left[2^{\ell} \int_{Q_{\ell, n}^{*}}\left|\frac{g_{12}}{\operatorname{det} \mathscr{G}}(p)\right| d p\right.} \\
&\left.\quad+\int_{Q_{i, n}^{*}}\left|g_{12}(p) \frac{\partial}{\partial u}\left(\frac{1}{\operatorname{det} \mathscr{G}}\right)(p)\right| d p\right] \\
& \leq C 2^{2 \ell}\left|Q_{\ell, n}^{*}\right| \leq C 2^{-\ell} .
\end{aligned}
$$

The estimate for $I I I$ is the same as for $I I$. We simply use (5.25) instead of (5.24). This completes the proof of (8.5) and so we have shown that $\rho_{t}^{1}$ satisfies (8.1).

\section{THE PROOF OF (1.2)-THE CONTRIBUTION FROM $\Omega_{k}^{2}$}

Let $\left\{\psi_{\ell}\right\}$ denote the functions from Lemma 7.1 which are supported in the rectangular parallelepipeds $D^{*}\left(p_{\ell}\right), p_{\ell} \in \Omega_{k}^{2}$. Then

$$
\int_{\Omega_{k}^{2}} f\left(\varphi_{t}(s, u, w)\right) d s d u d w=\int f(x) \rho_{t}^{2}(x) d x,
$$

where

$$
\rho_{t}^{2}(x)=\sum_{\ell} \frac{\psi_{\ell}\left(\varphi_{t}^{-1}(x)\right)}{\left|J_{\varphi_{t}}\left(\varphi_{t}^{-1}(x)\right)\right|} .
$$

We have to show

$$
\int\left|\rho_{t}^{2}(x \cdot y)-\rho_{t}^{2}(x)\right| d x \leq C h^{\epsilon}
$$

for $h<\|y\|$.

We let

$$
\rho_{\ell}(x)=\frac{\psi_{\ell}\left(\varphi_{t}^{-1}(x)\right)}{\left|J_{\varphi_{t}}\left(\varphi_{t}^{-1}(x)\right)\right|}
$$

and we shall show

$$
\begin{aligned}
& \int\left|\rho_{\ell}(x \cdot y)-\rho_{\ell}(x)\right| d x \leq C r_{1}\left(p_{\ell}\right) r_{2}\left(p_{\ell}\right) r_{3}\left(p_{\ell}\right), \\
& \int\left|\rho_{\ell}(x \cdot y)-\rho_{\ell}(x)\right| d x \leq C \frac{r_{2}\left(p_{\ell}\right)}{\lambda\left(2^{-k} s_{\ell}\right)}\|y\|,
\end{aligned}
$$

where $p_{\ell}=\left(s_{\ell}, u_{\ell}, w_{\ell}\right)$, and $\psi_{\ell}$ is supported in $D^{*}\left(p_{\ell}\right)$.

The proof of $(9.3)$ is immediate since

$$
\left|D^{*}\left(p_{\ell}\right)\right| \leq C r_{1}\left(p_{\ell}\right) r_{2}\left(p_{\ell}\right) r_{3}\left(p_{\ell}\right)
$$

We turn to the proof of (9.4). As before let

$$
K(s, u, w)=\frac{\psi_{\ell}(s, u, w)}{\left|J_{\varphi_{t}}(s, u, w)\right|} .
$$


Then as in the proof of (8.4), we see that the left-hand side of (9.4) is dominated by $\|y\|(I+I I+I I I)$ plus simpler integrals, where

$$
\begin{aligned}
I & =\int\left|g_{11}(s, u, w) \frac{\partial K}{\partial s}\right| d s d u d w, \\
I I & =\int\left|g_{12}(s, u, w) \frac{\partial K}{\partial u}\right| d s d u d w,
\end{aligned}
$$

and

$$
I I I=\int\left|g_{13}(s, u, w) \frac{\partial K}{\partial w}\right| d s d u d w .
$$

Hence to show (9.4), it suffices to show

$$
I+I I+I I I \leq C \frac{r_{2}\left(p_{\ell}\right)}{\lambda\left(2^{-k} s_{\ell}\right)} .
$$

Inequality (9.5) will follow from Lemma 5.9. First of all from (7.6), (5.27), (5.29), (5.31) and Lemma 5.8, we have

$$
\begin{gathered}
I \leq C \frac{1}{r_{1}\left(p_{\ell}\right)} \int_{D^{*}\left(p_{\ell}\right)}\left|\frac{g_{11}(p)}{\operatorname{det} \mathscr{G}(p)}\right| d p, \\
I I \leq C \frac{1}{r_{2}\left(p_{\ell}\right)} \int_{D^{*}\left(p_{\ell}\right)}\left|\frac{g_{12}}{\operatorname{det} \mathscr{G}}(p)\right| d p,
\end{gathered}
$$

and

$$
I I I \leq C \frac{1}{r_{3}\left(p_{\ell}\right)} \int_{D^{*}\left(p_{\ell}\right)}\left|\frac{g_{13}}{\operatorname{det} \mathscr{G}}(p)\right| d p .
$$

Now using (5.26), (5.28), (5.30) and Lemma 5.8, we see that the above integrals are each bounded by $\frac{r_{2}\left(p_{\ell}\right)}{\lambda\left(2^{-k} s_{\ell}\right)}$ and this gives (9.5). In the above analysis we used the fact that the function $\lambda\left(2^{-k}\right.$.) does not change much in $D^{*}\left(p_{\ell}\right)$. This follows from Lemmas 4.4 and 5.5. This completes the proof of $(9.4)$.

Inequalities (9.3) and (9.4) together imply for $0 \leq \delta \leq 1$,

$$
\begin{aligned}
& \int\left|\rho_{\ell}(x \cdot y)-\rho_{\ell}(x)\right| d x \leq C\left|D_{*}\left(p_{\ell}\right)\right|^{1-\delta}\left[\frac{r_{2}\left(p_{\ell}\right)}{\lambda\left(2^{-k} s_{\ell}\right)}\right]^{\delta}\|y\|^{\delta} \\
& \leq C\|y\|^{\delta} \int_{D_{*}\left(p_{\ell}\right)}\left[\frac{r_{2}\left(p_{\ell}\right)}{\left|D_{*}\left(p_{\ell}\right)\right| \lambda\left(2^{-k} s_{\ell}\right)}\right]^{\delta} d p \\
& \leq C\|y\|^{\delta} \int_{D_{*}\left(p_{\ell}\right)}\left[\frac{1}{r_{1}(p) r_{3}(p) \lambda\left(2^{-k} s\right)}\right]^{\delta} d p \\
& \leq C\|y\|^{\delta} \int_{D_{*}\left(p_{\ell}\right)} \lambda\left(2^{-k} w\right)^{\delta}\left[\frac{\gamma_{k}^{\prime}(s) \gamma_{k}^{\prime}(w)}{\left|J_{\varphi_{\ell}}(s, u, w)\right|}\right]^{2 \delta}\left[\frac{1}{\Delta(p)}\right]^{2 \delta} d s d u d w .
\end{aligned}
$$


Therefore

$$
\begin{aligned}
& \int\left|\rho_{t}^{2}(x \cdot y)-\rho_{t}^{2}(x)\right| d x \\
& \leq C\|y\|^{\delta} \int_{\Omega_{k}^{2}} \lambda\left(2^{-k} u\right)^{\delta}\left[\frac{\gamma_{k}^{\prime}(s) \gamma_{k}^{\prime}(w)}{\left|J_{\varphi_{t}}(s, u, w)\right|}\right]^{2 \delta} \frac{1}{|\Delta(s, u, w)|^{2 \delta}} d s d u d w \\
& \leq C\|y\|^{\delta} \int_{\Omega_{k}^{2}} \frac{1}{(w-u)^{\delta}} \frac{1}{|\Delta(s, u, w)|^{2 \delta}}\left[\frac{\gamma_{k}^{\prime}(s) \gamma_{k}^{\prime}(w)}{\left|J_{\varphi_{t}}(s, u, w)\right|}\right]^{2 \delta} d s d u d w .
\end{aligned}
$$

We used here the fact that for $p=(s, u, w) \in \Omega_{k}^{2}$,

$$
w-u \geq C \frac{\log \lambda\left(2^{-k} u\right)}{\lambda\left(2^{-k} u\right)} \geq C \frac{1}{\lambda\left(2^{-k} u\right)}
$$

if $k$ is large enough. Now we do the $s$ integration first and use Lemma 5.2 to estimate $J_{\varphi_{t}}$ from below and we obtain

$$
\begin{aligned}
& \int\left|\rho_{t}^{2}(x \cdot y)-\rho_{t}^{2}(x)\right| d x \\
& \quad \leq C\|y\|^{\delta} \iint_{\Omega_{k}^{2}} \frac{1}{(w-u)^{5 \delta}} d u d w \leq C\|y\|^{\delta}
\end{aligned}
$$

if $\delta$ is sufficiently small. This concludes the proof of $(9.2)$.

\section{THE PROOF OF (1.2)-THE CONTRIBUTION FROM $\Omega_{k}^{3}$}

Let $\left\{\psi_{\ell}\right\}$ denote the functions from Lemma 7.1 which are supported in the rectangular parallelepipeds $G^{*}\left(p_{\ell}\right), p_{\ell} \in \Omega_{k}^{3}$. Then we have

$$
\int_{\Omega_{k}^{3}} f\left(\varphi_{t}(s, u, w)\right) d s d u d w=\int f(x) \rho_{t}^{3}(x) d x
$$

where

$$
\rho_{t}^{3}(x)=\sum_{\ell} \frac{\psi_{\ell}\left(\varphi_{t}^{-1}(x)\right)}{\left|J_{\varphi_{t}}\left(\varphi_{t}^{-1}(x)\right)\right|} .
$$

We must show

$$
\int\left|\rho_{t}^{3}(x \cdot y)-\rho_{t}^{3}(x)\right| d x \leq C h^{\epsilon}
$$

for $\|y\|<h$.

We let

$$
\rho_{\ell}(x)=\frac{\psi_{\ell}\left(\varphi_{t}^{-1}(x)\right)}{\left|J_{\varphi_{t}}\left(\varphi_{t}^{-1}(x)\right)\right|} .
$$

We wish to show

$$
\int\left|\rho_{\ell}(x \cdot y)-\rho_{\ell}(x)\right| d x \leq C \tilde{r}_{1}\left(p_{\ell}\right) \tilde{r}_{2}\left(p_{\ell}\right) \tilde{r}_{3}\left(p_{\ell}\right),
$$


and

$$
\int\left|\rho_{\ell}(x \cdot y)-\rho_{\ell}(x)\right| d x \leq C\|y\| \tilde{r}_{2}\left(p_{\ell}\right) .
$$

Again (10.3) is trivial. Furthermore, in analogy with Sections 8 and 9, the main work in proving $(10.4)$ is the estimate

$$
I+I I+I I I \leq C \tilde{r}_{2}\left(p_{\ell}\right)
$$

where

$$
\begin{gathered}
I=\int_{G^{*}\left(p_{\ell}\right)}\left|g_{11}(p) \frac{\partial K}{\partial s}\right| d p, \\
I I=\int_{G^{*}\left(p_{\ell}\right)}\left|g_{12}(p) \frac{\partial K}{\partial u}\right| d p,
\end{gathered}
$$

and

$$
I I I=\int_{G^{*}\left(p_{\ell}\right)}\left|g_{13}(p) \frac{\partial K}{\partial w}\right| d p .
$$

As in Section 9, we can use (7.7), (5.33), (5.35), (5.37) and Lemma 5.8 to show

$$
\begin{gathered}
I \leq C \frac{1}{\tilde{r}_{1}\left(p_{\ell}\right)} \int_{G^{*}\left(p_{\ell}\right)}\left|\frac{g_{11}(p)}{\operatorname{det} \mathscr{G}(p)}\right| d p, \\
I I \leq C \frac{1}{\tilde{r}_{2}\left(p_{\ell}\right)} \int_{G^{*}\left(p_{\ell}\right)}\left|\frac{g_{12}}{\operatorname{det} \mathscr{G}}(p)\right| d p,
\end{gathered}
$$

and

$$
I I I \leq C \frac{1}{\tilde{r}_{3}\left(p_{\ell}\right)} \int_{G^{*}\left(p_{\ell}\right)}\left|\frac{g_{13}}{\operatorname{det} \mathscr{G}}(p)\right| d p .
$$

Finally using (5.32), (5.34), (5.36) and Lemma 5.8 gives us (10.5). This completes the proof of (10.4).

We now complete the proof of (10.2) in analogy with Section 9. From (10.3) and (10.4), we see that for every $0<\delta \leq 1$,

$$
\begin{aligned}
& \int_{G^{*}\left(p_{\ell}\right)}\left|\rho_{\ell}(x \cdot y)-\rho_{\ell}(x)\right| d x \leq C\left|G_{*}\left(p_{\ell}\right)\right|^{1-\delta}\left(\tilde{r}_{2}\left(\rho_{\ell}\right)\|y\|\right)^{\delta} \\
& \leq C\|y\|^{\delta} \int_{G_{*}\left(p_{\ell}\right)}\left[\frac{1}{\tilde{r}_{1}\left(p_{\ell}\right) \tilde{r}_{3}\left(p_{\ell}\right)}\right]^{\delta} d p \\
& \leq C\|y\|^{\delta} \int_{G_{*}\left(p_{\ell}\right)} \lambda\left(2^{-k} s\right)^{\delta}\left|\frac{\gamma_{k}^{\prime}(s) \gamma_{k}^{\prime}(w)}{J_{\varphi_{t}}(p) \Delta(p)}\right|^{2 \delta} d p .
\end{aligned}
$$

Summing on $\ell$ gives us

$$
\begin{aligned}
& \int\left|\rho_{t}^{3}(x \cdot y)-\rho_{t}^{3}(x)\right| d x \\
& \leq C\|y\|^{\delta} \int_{\Omega_{k}^{3}} \lambda\left(2^{-k} s\right)^{\delta}\left|\frac{\gamma_{k}^{\prime}(s) \gamma_{k}^{\prime}(w)}{J_{\varphi_{t}}(p) \Delta(p)}\right|^{2 \delta} d p \\
& \leq C\|y\|^{\delta} \int_{\Omega_{k}^{3}} \frac{1}{\left|P_{t}(s, u, w)\right|^{\delta}}\left|\frac{\gamma_{k}^{\prime}(s) \gamma_{k}^{\prime}(w)}{J_{\varphi_{t}}(p) \Delta(p)}\right|^{2 \delta} d p
\end{aligned}
$$


since for $p=(s, u, w) \in \Omega_{k}^{3},\left|P_{t}(s, u, w)\right| \geq C \frac{1}{\lambda\left(2^{-k} s\right)}$. Doing the $s$ integral first and using Lemma 5.2 to estimate $J_{\varphi_{t}}$ from below now gives us (10.2) if we choose $\delta$ above to be sufficiently small. This completes the proof of (1.2) and (1.3).

\section{The $L^{p}$ BOUNDEDNESS OF $\mathscr{M} f$}

Let $d \nu_{k}$ be the measure which acts on a test function by

$$
d \nu_{k}(f)=2^{k} \int_{2^{-k}}^{2^{-k+1}} f(\Gamma(t)) d t
$$

With the notation introduced in Section 1 we see that $d \nu_{k}$ is simply the measure such that $M_{k} f(x)=f * d \nu_{k}(x)$. Also in Section 1 we saw that the $L^{p}$ estimates for $\mathscr{M} f$ follow from

$$
\|M f\|_{L^{p}} \leq C_{p}\|f\|_{L^{p}}, \quad 1<p \leq \infty,
$$

where $M f(x)=\sup _{k>0}\left|M_{k} f(x)\right|$.

We will prove (11.1) by following the bootstrap argument in [NSW1]. That is, we shall prove the following three lemmas.

Lemma 11.1. $M$ is bounded on $L^{2}\left(\mathbb{R}^{3}\right)$.

Lemma 11.2. Suppose that for some $p_{0}<2$,

$$
\left\|\left(\sum_{k}\left|f_{k} * d \nu_{k}\right|^{2}\right)^{1 / 2}\right\|_{L^{p_{0}}} \leq C_{p_{0}}\left\|\left(\sum_{k}\left|f_{k}\right|^{2}\right)^{1 / 2}\right\|_{L^{p_{0}}} ;
$$

then

$$
\|M f\|_{L^{p}} \leq C_{p}\|f\|_{L^{p}}, \quad p_{0}<p \leq 2 .
$$

Lemma 11.3. Suppose that for some $p_{0} \leq 2$,

$$
\|M f\|_{L^{p_{0}}} \leq C_{p_{0}}\|f\|_{L^{p_{0}}}
$$

then

$$
\left\|\left(\sum_{k}\left|f_{k} * d \nu_{k}\right|^{2}\right)^{1 / 2}\right\|_{L^{p}} \leq C_{p}\left\|\left(\sum_{k}\left|f_{k}\right|^{2}\right)^{1 / 2}\right\|_{L^{p}}
$$

for all $p$ with $\frac{1}{p} \leq \frac{1}{2}\left(\frac{1}{p_{0}}+1\right)$.

The proof of Lemma 11.3 is given in [NSW1], and so we concentrate on the proofs of Lemmas 11.1 and 11.2. We begin by recalling some known results. Let

$$
\delta(t)=\left(\begin{array}{ccc}
t & 0 & 0 \\
0 & \gamma(t) & 0 \\
0 & 0 & t \gamma(t)
\end{array}\right) .
$$

The convexity of $\gamma$ implies

$$
\left\|\delta^{-1}(t) \delta(s)\right\| \leq s / t
$$


if $s<t$. We also put $A_{k}=\delta\left(2^{-k}\right)$ and note that

$$
\left\|A_{k}^{-1} A_{k+1}\right\| \leq 1 / 2 \text {. }
$$

Let $\psi \in C_{0}^{\infty}\left(\mathbb{R}^{3}\right)$ with $\int_{\mathbb{R}^{3}} \psi=1$ and $\psi(x)=\psi(-x)$. Set

$$
\Psi_{k}(x)=\frac{1}{\operatorname{det} A_{k+1}} \psi\left(A_{k+1}^{-1} x\right)-\frac{1}{\operatorname{det} A_{k}} \psi\left(A_{k}^{-1} x\right) .
$$

Then we have the following Littlewood-Paley inequality,

$$
\left\|\left(\sum_{k}\left|f * \Psi_{k}\right|^{2}\right)^{1 / 2}\right\|_{L^{p}} \leq C_{p}\|f\|_{L^{p}}, \quad 1<p<\infty .
$$

See [CVWW].

Now let $\varphi$ be a second $C_{0}^{\infty}$ function on $\mathbb{R}^{3}$ with $\varphi \geq 0, \int \varphi=1$, and $\varphi(x)=\varphi(-x)$. Set

$$
\varphi_{k}(x)=\frac{1}{\operatorname{det} A_{k}} \varphi\left(A_{k}^{-1} x\right),
$$

and $N f(x)=\sup _{k}\left|f * \varphi_{k}(x)\right|$. Then according to [CVWW],

$$
\|N f\|_{L^{p}} \leq C_{p}\|f\|_{L^{p}}, \quad 1<p \leq \infty .
$$

Furthermore the argument in [NSW] proving Lemma 11.3 shows

$$
\left\|\left(\sum_{k}\left|f_{k} * \varphi_{k}\right|^{2}\right)^{1 / 2}\right\|_{L^{p}} \leq C_{p}\left\|\left(\sum_{k}\left|f_{k}\right|^{2}\right)^{1 / 2}\right\|_{L^{p}}
$$

$1<p \leq 2$. In view of (11.6), (11.1) will follow from the inequality

$$
\left\|\sup _{k>0}\left|f *\left(d \nu_{k}-\varphi_{k}\right)\right|\right\|_{L^{p}} \leq C_{p}\|f\|_{L^{p}}
$$

Now

$$
f *\left(d \nu_{k}-\varphi_{k}\right)(x)=\sum_{\ell} f * \Psi_{k+\ell} *\left(d \nu_{k}-\varphi_{k}\right)(x)
$$

and so

$$
\sup _{k>0}\left|f *\left(d \nu_{k}-\varphi_{k}\right)(x)\right| \leq \sum_{\ell}\left(\sum_{k \geq 0}\left|f * \Psi_{k+\ell} *\left(d \nu_{k}-\varphi_{k}\right)\right|^{2}\right)^{1 / 2}
$$

Set

$$
G_{\ell} f(x)=\left(\sum_{k \geq 0}\left|f * \Psi_{k+\ell} *\left(d \nu_{k}-\varphi_{k}\right)(x)\right|^{2}\right)^{1 / 2}
$$

We shall show that for some $\epsilon>0$,

$$
\left\|G_{\ell} f\right\|_{L^{2}} \leq C 2^{-\epsilon|\ell|}\|f\|_{L^{2}} .
$$


This will then prove Lemma 11.1. Then under the hypothesis of Lemma 11.2, using (11.7), we see

$$
\left\|G_{\ell} f\right\|_{L^{p_{0}}} \leq C_{p_{0}}\left\|\left(\sum_{k \geq 0}\left|f * \Psi_{k+\ell}\right|^{2}\right)\right\|_{L^{p_{0}}} .
$$

Now using (11.5), we obtain

$$
\left\|G_{\ell} f\right\|_{L^{p_{0}}} \leq C_{p_{0}}\|f\|_{L^{p_{0}}}
$$

Interpolating with (11.9), we find

$$
\left\|G_{\ell} f\right\|_{L^{p}} \leq C 2^{-\epsilon(p)|\ell|}\|f\|_{L^{p}}, \quad p_{0}<p \leq 2,
$$

for some $\epsilon(p)>0$. Summing on $\ell$ then completes the proof of Lemma 11.2.

Thus matters are reduced to proving (11.9). Let $r_{k}(t)$ denote the standard Rademacher functions, and set

$$
T_{\ell, t} f(x)=\sum_{k} r_{k}(t) f * \Psi_{k+\ell} *\left(d \nu_{k}-\varphi_{k}\right)(x) .
$$

A standard argument shows that (11.9) will follow from

$$
\left\|T_{\ell, t} f\right\|_{L^{2}} \leq C 2^{-\epsilon|\ell|}\|f\|_{L^{2}}
$$

Let

We will show

$$
S_{k}^{\ell} f(x)=f * \Psi_{k+\ell} *\left(d \nu_{k}-\varphi_{k}\right)(x)
$$

$$
\left\|S_{k}^{\ell}\left(S_{j}^{\ell}\right)^{*}\right\| \leq C 2^{-\epsilon|\ell|} 2^{-\delta|j-k|}
$$

for some $\epsilon>0$ and $\delta>0$. Here $\|\cdot\|$ denotes the operator norm on $L^{2}$. A similar but somewhat more complicated argument shows

$$
\left\|\left(S_{k}^{\ell}\right)^{*} S_{j}^{\ell}\right\| \leq C 2^{-\epsilon|\ell|} 2^{-\delta|j-k|} .
$$

Then (11.12) and (11.13) imply (11.11) by the Cotlar-Stein lemma.

Note that

$$
S_{k}^{\ell}\left(S_{j}^{\ell}\right)^{*} f=f *\left(d \nu_{j}^{*}-\varphi_{j}\right) * \Psi_{j+\ell} * \Psi_{k+\ell} *\left(d \nu_{k}-\varphi_{k}\right),
$$

where $d \nu_{j}^{*}$ is the measure defined by

$$
d \nu_{j}^{*}(g)=2^{j} \int_{2^{-j}}^{2^{-j+1}} g\left(\Gamma^{-1}(t)\right) d t
$$

Since the total mass of $d \nu_{k}$ and $\varphi_{k}$ is bounded, we have

$$
\left\|S_{k}^{\ell}\left(S_{j}^{\ell}\right)^{*}\right\| \leq C\left\|\Psi_{j+\ell} * \Psi_{k+\ell}\right\|_{L^{\prime}} \leq C 2^{-\epsilon|j-k|} \text {. }
$$

To verify the last inequality, we may assume $j \leq k$ and note that since $\Psi_{j+\ell}$ has mean value zero,

$$
\left\|\Psi_{j+\ell} * \Psi_{k+\ell}\right\|_{L^{1}} \leq C \int_{\mathbb{R}^{3}}\left|\Psi_{j+\ell}(x)\left\|A_{k+\ell}^{-1} x \mid d x \leq C\right\| A_{k+\ell}^{-1} A_{j+\ell} \| \leq C 2^{-(k-j)}\right.
$$

by $(11.3)$. 
Therefore it remains to show

$$
\left\|S_{k}^{\ell}\left(S_{j}^{\ell}\right)^{*}\right\| \leq C 2^{-\epsilon|\ell|} .
$$

We shall first prove (11.14) when $\ell \leq 0$. Note that

$$
\left\|S_{k}^{\ell}\right\| \leq C\left\|\Psi_{k+\ell} *\left(d \nu_{k}-\varphi_{k}\right)\right\|_{L^{1}}
$$

As before since $d \nu_{k}-\varphi_{k}$ has mean value zero, we have

$$
\begin{aligned}
\| \Psi_{k+\ell} & *\left(d \nu_{k}-\varphi_{k}\right) \|_{L^{1}} \\
& \leq C\left[\int_{\mathbb{R}^{3}}\left|A_{k+\ell}^{-1} x\right| d \nu_{k}(x)+\int_{\mathbb{R}^{3}}\left|A_{k+\ell}^{-1} x\right| \varphi_{k}(x) d x\right] \\
& \leq C 2^{k} \int_{2^{-k}}^{2^{-k+1}}\left|A_{k+\ell}^{-1} \Gamma(t)\right| d t+C\left\|A_{k+\ell}^{-1} A_{k}\right\| \\
& =C \int_{1 / 2}^{1}\left|A_{k+\ell}^{-1} A_{k-1} \Gamma_{k-1}(t)\right| d t+C\left\|A_{k+\ell}^{-1} A_{k}\right\| \\
& \leq C\left\|A_{k+\ell}^{-1} A_{k}\right\| \leq C 2^{\ell} .
\end{aligned}
$$

Here we used the fact that the normalized curve $\Gamma_{k-1}(t)$ is bounded for $\frac{1}{2} \leq$ $t \leq 1$. This proves (11.14) for $\ell<0$.

We now suppose $\ell>0$. Write

$$
\begin{aligned}
S_{k}^{\ell} f & =f * \Psi_{k+\ell} * d \nu_{k}-f * \Psi_{k+\ell} * \varphi_{k} \\
& =R_{k}^{\ell} f-Q_{k}^{\ell} f .
\end{aligned}
$$

We shall show

$$
\left\|R_{k}^{\ell} f\right\|_{L^{2}} \leq C 2^{-\epsilon \ell}\|f\|_{L^{2}}
$$

The estimate for $Q_{k}^{\ell}$ is easier. Since

$$
\begin{gathered}
\left(R_{k}^{\ell}\right)^{*} R_{k}^{\ell} f(x)=f * \Psi_{k+\ell} * d \nu_{k} * d \nu_{k}^{*} * \Psi_{k+\ell}(x), \\
\left\|\left(R_{k}^{\ell}\right)^{*} R_{t}^{\ell} f\right\|_{L^{2}} \leq C\left\|f * \Psi_{k+\ell} * d \nu_{k} * d \nu_{k}^{*}\right\|_{L^{2}} .
\end{gathered}
$$

Let $\bar{R} f(x)=f * \Psi_{k+\ell} * d \nu_{k} * d \nu_{k}^{*}(x)$.

$$
\begin{aligned}
\left\|\bar{R}^{*} \bar{R} f\right\|_{L^{2}} & =\left\|f * \Psi_{k+\ell} * d \nu_{k} * d \nu_{k}^{*} * d \nu_{k} * d \nu_{k}^{*} * \Psi_{k+\ell}\right\|_{L^{2}} \\
& \leq C\left\|\Psi_{k+\ell} * d \nu_{k} * d \nu_{k}^{*} * d \nu_{k} * d \nu_{k}^{*} * \Psi_{k+\ell}\right\|_{L^{1}}\|f\|_{L^{2}}
\end{aligned}
$$

Therefore since

$$
\left\|R_{k}^{\ell}\right\|=\left\|\left(R_{k}^{\ell}\right)^{*} R_{k}^{\ell}\right\|^{1 / 2} \leq\|\bar{R}\|^{1 / 2} \leq\left\|\bar{R}^{*} \bar{R}\right\|^{1 / 4},
$$

it suffices to show

$$
\left\|\Psi_{k+\ell} * d \nu_{k} * d \nu_{k}^{*} * d \nu_{k} * d \nu_{k}^{*} * \Psi_{k+\ell}\right\|_{L^{1}} \leq C 2^{-\epsilon \ell}
$$

for some $\epsilon>0$. We know from Section 1 that

$$
\begin{aligned}
\Psi_{k+\ell} & * d \nu_{k} * d \nu_{k}^{*} * d \nu_{k} * d \nu_{k}^{*}(x) \\
& =\Psi_{k+\ell, k} * d \mu_{k} * d \mu_{k}^{*} * d \mu_{k} * d \mu_{k}^{*}\left(A_{k}^{-1} x\right)
\end{aligned}
$$


where $\Psi_{k+\ell, k}(x)=\Psi_{k+\ell}\left(A_{k} x\right)$ and $d \mu_{k}$ is the normalized measure along the curve $\Gamma(t)$. Similarly we have

$$
\begin{array}{rl}
d \nu_{k} & * d \nu_{k}^{*} * d \nu_{k} * d \nu_{k}^{*} * \Psi_{k+\ell}(x) \\
& =d \mu_{k} * d \mu_{k}^{*} * d \mu_{k} * d \mu_{k}^{*} * \Psi_{k+\ell, k}\left(A_{k}^{-1} x\right) .
\end{array}
$$

Also from Sections 3 to 10 , we know that

$$
d \mu_{k} * d \mu_{k}^{*} * d \mu_{k} * d \mu_{k}^{*}=\left(\rho_{k, 1}(x)+\rho_{k, 2}(x)\right) d x
$$

where

$$
\int\left|\rho_{k, 1}(x \cdot y)-\rho_{k, 1}(x)\right| d x \leq C h^{\epsilon}
$$

if $\|y\|<h$, and

$$
\int\left|\rho_{k, 2}(y \cdot x)-\rho_{k, 2}(x)\right| d x \leq C h^{\epsilon}
$$

if $\|y\|<h$. Therefore we may write

$$
\begin{gathered}
\Psi_{k+\ell} * d \nu_{k} * d \nu_{k}^{*} * d \nu_{k} * d \nu_{k}^{*} * \Psi_{k+\ell} \\
\Psi_{k+\ell} * A+B * \Psi_{k+\ell}
\end{gathered}
$$

where

$$
A=\rho_{k, 1} * \Psi_{k+\ell, k}\left(A_{k}^{-1} x\right)
$$

and

$$
B=\Psi_{k+\ell, k} * \rho_{k, 2}\left(A_{k}^{-1} x\right)
$$

Thus it suffices to show

$$
\|A\|_{L^{1}} \leq C 2^{-\epsilon \ell}
$$

and

$$
\|B\|_{L^{1}} \leq C 2^{-\epsilon \ell}
$$

We will prove (11.16). The proof of $(11.15)$ is similar.

$$
\begin{aligned}
B & =\int \Psi_{k+\ell}\left(A_{k}\left(A_{k}^{-1} x \cdot y^{-1}\right)\right) \rho_{k, 2}(y) d y \\
& =\int \Psi_{k+\ell}\left(x \cdot\left(A_{k} y\right)^{-1}\right) \rho_{k, 2}(y) d y \\
& =\frac{1}{\operatorname{det} A_{\dot{k}}} \int \Psi_{k+\ell}(y) \rho_{k, 2}\left(A_{k}^{-1} y \cdot A_{k}^{-1} x\right) d y \\
& =\frac{1}{\operatorname{det} A_{k}} \int\left[\rho_{k, 2}\left(A_{k}^{-1} y \cdot A_{k}^{-1} x\right)-\rho_{k, 2}\left(A_{k}^{-1} x\right)\right] \Psi_{k+\ell}(y) d y
\end{aligned}
$$


since $\Psi_{k+\ell}$ has mean value zero. Therefore

$$
\begin{aligned}
\|B\|_{L^{1}} \leq & \frac{1}{\operatorname{det} A_{k}} \int\left|\Psi_{k+\ell}(y)\right|\left[\int\left|\rho_{k, 2}\left(A_{k}^{-1} y \cdot A_{k}^{-1} x\right)-\rho_{k, 2}\left(A_{k}^{-1} x\right)\right| d x\right] d y \\
= & \int\left|\Psi_{k+\ell}(y)\right|\left[\int\left|\rho_{k, 2}\left(A_{k}^{-1} y \cdot x\right)-\rho_{k, 2}(x)\right| d x\right] d y \\
\leq & C \int\left|\Psi_{k+\ell}(y)\right|\left\|A_{k}^{-1} y\right\|^{\epsilon} d y \\
\leq & C \int|\psi(y)|\left\|A_{k}^{-1} A_{k+\ell+1} y\right\|^{\epsilon} d y \\
& +C \int|\psi(y)|\left\|A_{k}^{-1} A_{k+\ell} y\right\|^{\epsilon} d y \\
\leq & C 2^{-\epsilon \ell}
\end{aligned}
$$

The last inequality follows from (11.3) and this completes the proof of (11.16), finishing the proof of the $L^{p}$ boundedness of $\mathscr{M} f$.

\section{NOTE ADDED IN PROOF}

$L^{2}$ estimates for related, but different, singular Radon transforms were recently obtained by A. Seeger using completely different methods. His article is entitled $L^{2}$-estimates for a class of singular oscillatory integrals, and appears in Math. Res. Lett. I (1994), 65-73.

\section{REFERENCES}

[CVWWA] A. Carbery, J. Vance, S. Wainger, and D. Watson, The Hilbert transform and maximal function along flat curves, dilations and differential equations, Amer. J. Math. (to appear).

[CVWWW] A. Carbery, J. Vance, S. Wainger, D. Watson, and J. Wright, $L^{p}$ estimates for operators associated to flat curves without the Fourier transform, Pacific J. Math (to appear).

[CVWW] A. Carbery, J. Vance, S. Wainger, and J. Wright, A variant of the notion of a space of homogeneous type (to appear).

[CZ] A. Carbery and S. Ziesler, Hilbert transforms and maximal functions along rough flat curves (to appear).

[C1] M. Christ, Hilbert transforms along curves, III. Rotational curvature, preprint, 1985.

[C2] Hilbert transforms along curves, I. Nilpotent groups, Ann. of Math. (2) 122 (1985), 575-596.

[CNSW] M. Christ, A. Nagel, E. Stein, and S. Wainger, Singular and maximal Radon transforms, Ann. of Math. Stud., Princeton Univ. Press, Princeton, NJ (to appear).

[CW] R. Coifman and G. Weiss, Analyse harmonique non-commutative sur certains espaces homogenes, Lectures Notes in Math., vol. 242, Springer-Verlag, Berlin and New York, 1971.

[NSW] A. Nagel, E. Stein, and S. Wainger, Hilbert transforms and maximal functions related to variable curves, Proc. Sympos. Pure Math., vol. 35, Part 2, Amer. Math. Soc., Providence, RI, 1979, pp. 95-98.

[NSW1] A. Nagel, E. Stein, and S. Wainger, Differentiation in lacunary directions, Proc. Nat. Acad. Sci. U.S.A. 75 (1978), 1060-1062.

[RS] F. Ricci and E. Stein, Harmonic analysis on nilpotent groups and singular integrals, III. Kernels supported on submanifolds, J. Funct. Anal. 78 (1988), 56-84. 
[S] E. Stein, Singular integrals and differentiability properties of functions, Princeton Univ. Press, Princeton, NJ, 1970.

[SS] C. Sogge and E. Stein, Averages of functions over hypersurfaces in $\mathbb{R}^{n}$, Invent. Math. 82 (1985), 543-556.

ICMS, 14 India Stree, Edinburgh, EH3 6EZ, Scotland 